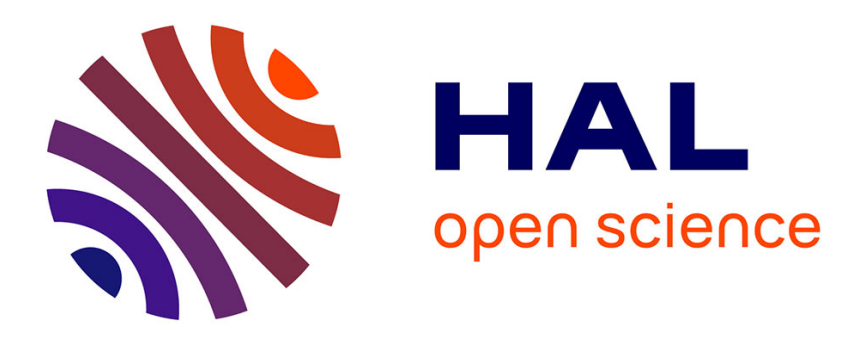

\title{
Tits' type alternative for groups acting on toric affine varieties
}

Ivan Arzhantsev, M Zaidenberg

\section{To cite this version:}

Ivan Arzhantsev, M Zaidenberg. Tits' type alternative for groups acting on toric affine varieties. 2020. hal-02494577v4

\author{
HAL Id: hal-02494577 \\ https://hal.science/hal-02494577v4
}

Preprint submitted on 4 Nov 2020

HAL is a multi-disciplinary open access archive for the deposit and dissemination of scientific research documents, whether they are published or not. The documents may come from teaching and research institutions in France or abroad, or from public or private research centers.
L'archive ouverte pluridisciplinaire HAL, est destinée au dépôt et à la diffusion de documents scientifiques de niveau recherche, publiés ou non, émanant des établissements d'enseignement et de recherche français ou étrangers, des laboratoires publics ou privés. 


\section{Tits' type alternative for groups acting on toric affine varieties}

\section{Ivan Arzhantsev ${ }^{1}$ and Mikhail Zaidenberg ${ }^{2}$}

${ }^{1}$ National Research University Higher School of Economics, Faculty of Computer Science, Pokrovsky Boulevard 11, Moscow, 109028 Russia and ${ }^{2}$ Université Grenoble Alpes, CNRS, Institut Fourier, F-38000 Grenoble, France

Correspondence to be sent to: Mikhail.Zaidenberg@univ-grenoble-alpes.fr

Given a toric affine algebraic variety $X$ and a collection of one-parameter unipotent subgroups $U_{1}, \ldots, U_{s}$ of $\operatorname{Aut}(X)$ which are normalized by the torus acting on $X$, we show that the group $G$ generated by $U_{1}, \ldots, U_{s}$ verifies the following alternative of Tits' type: either $G$ is a unipotent algebraic group, or it contains a non-abelian free subgroup. We deduce that if $G$ is 2-transitive on a $G$-orbit in $X$, then $G$ contains a non-abelian free subgroup, and so, is of exponential growth.

\section{Introduction}

We fix an algebraically closed field $\mathbf{k}$ of characteristic zero. Let $\mathbb{A}^{n}$ stand for the affine space of dimension $n$ over $\mathbf{k}$ and $\mathbb{G}_{a}\left(\mathbb{G}_{m}\right)$ for the additive (multiplicative, respectively) group of $\mathbf{k}$ viewed as an algebraic group. Consider an algebraic variety $X$ over $\mathbf{k}$ and an effective regular action $\mathbb{G}_{a} \times X \rightarrow X$. The image of $\mathbb{G}_{a}$ in Aut $(X)$ is called a one-parameter unipotent subgroup of $\operatorname{Aut}(X)$, or a $\mathbb{G}_{a}$-subgroup, for short. Any $\mathbb{G}_{a}$-subgroup $U$ of Aut $(X)$ has the form $U=\{\exp (t \partial) \mid t \in \mathbf{k}\}$, where $\partial$ is a locally nilpotent derivation of the structure $\operatorname{ring} \mathcal{O}(X)$. This correspondence between the $\mathbb{G}_{a}$-subgroups and locally nilpotent derivations does not hold in prime characteristic, and so, we prefer in this paper to work in characteristic zero.

The main result of the paper is the following

Theorem 1.1. Consider a toric affine variety $X$ with no torus factor. Let a subgroup $G$ of Aut $(X)$ be generated by a finite collection $U_{1}, \ldots, U_{s}$ of one-parameter unipotent subgroups normalized by the acting torus. Then either

(i) $G$ is a unipotent algebraic group, or

(ii) $G$ contains the free group $\mathbf{F}_{2}$ of rank two as a subgroup.

One says that a variety $X$ over $\mathbf{k}$ has a torus factor if $X \cong Y \times(\mathbf{k} \backslash\{\mathbf{0}\})$ for some variety $Y$. A toric affine variety $X$ has a torus factor if and only if there is a nonconstant invertible regular function on $X$.

From Theorem 1.1 we deduce the following corollary.

Corollary 1.2. Let $G$ be a group acting on a toric affine variety $X$ and generated by a finite collection $U_{1}, \ldots, U_{s}$ of one-parameter unipotent subgroups normalized by the acting torus. If $G$ is doubly transitive on a $G$-orbit in $X$, then $G$ contains a free subgroup of rank two.

The expression "Tits' type alternative" in the present paper addresses a property of a class of groups which asserts that any group from this class either is virtually solvable (resp., virtually nilpotent, virtually abelian, etc.), or contains a non-abelian free subgroup. This (rather weak) form of the original Tits alternative disregards whether or not the alternative remains true when passing to a subgroup. We wonder whether, under the setup of Theorem 1.1, any (finitely generated) subgroup $H$ of $G$ either is virtually solvable, or contains a free subgroup of rank two. Notice that the group $G$ in Theorem 1.1] is not finitely generated, in general. 
Let us provide a brief survey on the classical Tits alternative for the automorphism groups arising in algebraic geometry. Recall that, due to the original Tits' theorem [Tits, 1972, Cor. 1], any finitely generated subgroup of a linear algebraic group either is virtually solvable, or contains a non-abelian free subgroup. Over a field of characteristic zero, the Tits alternative holds for any, not necessarily finitely generated, linear group Tits, 1972, Thm. 1]. In the sequel we call the latter property the enhanced Tits alternative. As examples, we can cite the following results. The first of them is due to S. Cantat and Ch. Urech.

Theorem 1.3. The group of birational transformations of a compact complex Kähler surface verifies the enhanced Tits alternative, that is, any of its non-virtually solvable subgroups contains a free subgroup of rank two.

The theorem is proven in [Cantat, 2011a, Thm. C and Sec. 6] (see also Cantat, 2011b, Prop. 6.3]) for finitely generated subgroups, and in full generality for irrational surfaces. In [Urech, 2020, Thm. 1.7 and Sec. 7] it is extended to the rational surfaces by showing that the Cremona group of the plane verifies the enhanced Tits alternative.

This theorem extends the earlier result of S. Lamy Lamv, 2001] which says that Aut $\left(\mathbb{A}_{\mathbb{C}}^{2}\right)$ verifies the enhanced Tits alternative. The enhanced Tits alternative holds also for the tame automorphism group of $\mathrm{SL}_{2}(\mathbb{C})$ viewed as an affine quadric threefold [Bisi et al., 2014, Thm. C], and as well for the group of birational transformations of any hyperkähler variety [Oguiso, 2006]; see also [Kurnosov et al., 2019]. Otherwise, its validity for the groups of birational transformations in higher dimensions is widely open. It is open, for instance, for the $\operatorname{group} \operatorname{Aut}\left(\mathbb{A}_{\mathbb{C}}^{3}\right)$; see also the survey article [Cantat, 2018.

As a nice application of the classical Fujiki-Lieberman theorem, it is shown in Cantat, 2011a, Thm. 6.3] that the enhanced Tits alternative holds for the automorphism group of any compact Kähler manifold. The geometry of the Kähler manifold controls the structure of the group when it is virtually solvable Campana et al., 2014, Thm. 1.5], Dinh, 2012], Dinh et al., 2015, Thm. 1.1]; see also [Hu, 2019] for the case of projective varieties over a field of positive characteristic.

Our starting point in the present work was actually the transitivity issue, see Corollary 1.2. Let $X$ be a toric affine variety over $\mathbf{k}$ of dimension at least two with no torus factor, and let $\operatorname{SAut}(X) \subset \operatorname{Aut}(X)$ be the subgroup generated by all the $\mathbb{G}_{a}$-subgroups of $\operatorname{Aut}(X)$. It is known Arzhantsev et al., 2012, Thm. 2.1] that $\operatorname{SAut}(X)$ acts highly transitively $*$ on the smooth $\operatorname{locus} \operatorname{reg}(X)$, that is, $m$-transitively for any $m \geq 1$. A variety $X$ satisfying the latter property is called flexible; see [Arzhantsev et al., 2013, Thm. 1.1] for a criterion of flexibility. Notice that an algebraic subgroup $G \subset \operatorname{Aut}(X)$ cannot act highly transitively on a variety, by a dimension count argument.

A $\mathbb{G}_{a}$-subgroup acting on a toric variety $X$ is called a root subgroup if it is normalized by the acting torus. The term root subgroup is due to the fact that any such subgroup is associated with a certain lattice vector called a Demazure root, see subsection 2.2. Assuming in addition that a toric affine variety $X$ is smooth in codimension two, one can find a finite number of root subgroups $U_{1}, \ldots, U_{s}$ of $\operatorname{Aut}(X)$ such that the group $G=\left\langle U_{1}, \ldots, U_{s}\right\rangle$ generated by these subgroups still acts highly transitively on $\operatorname{reg}(X)$ Arzhantsev et al., 2019, Thm. 1.1]. If If $X=\mathbb{A}^{n}, n \geq 2$, then just three $\mathbb{G}_{a}$-subgroups (which are not root subgroups, in general) are enough [Arzhantsev et al., 2019, Thm. 1.3]; such subgroups are found explicitly in [Andrist, 2019]. For instance, for $n=2$ the group $G$ generated by the root subgroups

$$
U_{1}=\left\{(x, y) \mapsto\left(x+t_{1} y^{2}, y\right)\right\} \quad \text { and } \quad U_{2}=\left\{(x, y) \mapsto\left(x, y+t_{2} x\right)\right\}, \quad t_{1}, t_{2} \in \mathbf{k}
$$

acts highly transitively on $\mathbb{A}^{2} \backslash\{0\}$ equipped with the standard action of the 2-torus, see Lewis et al., 2018, Cor. 21]. Adding one more root subgroup

$$
U_{3}=\left\{(x, y) \mapsto\left(x+t_{3}, y\right)\right\}, \quad t_{3} \in \mathbf{k}
$$

one gets the group $\left\langle U_{1}, U_{2}, U_{3}\right\rangle$ acting highly transitively on $\mathbb{A}^{2}$ (cf. Chistopolskava, 2018]).

The following question arises: What can one say about a group acting highly transitively on an algebraic variety? More specifically, let us formulate the following conjecture.

Conjecture 1. Let $X$ be an affine variety over $\mathbf{k}$ of dimension $\geq 2$. Consider the group

$$
G=\left\langle U_{1}, \ldots U_{s}\right\rangle
$$

generated by $\mathbb{G}_{a}$-subgroups $U_{1}, \ldots U_{s}$ of $\operatorname{Aut}(X)$. Suppose $G$ is doubly transitive on a $G$-orbit. Then $G$ contains a non-abelian free subgroup.

*Or infinitely transitively, in the terminology of [Arzhantsev et al., 2013].

${ }^{\dagger}$ It is conjectured [Arzhantsev et al., 2019, Conj. 1.1] that an analogous result holds for any flexible affine variety. 
Corollary 1.2 partially confirms Conjecture1. Of course, an analog of this conjecture makes sense in different categories. For instance, one might ask (following a referee's suggestion) whether any highly transitive group of homeomorphisms of a compact manifold contains a non-abelian free subgroup; see, e.g., Whittaker, 1967. for examples of highly transitive groups of homeomorphisms. However, the group-combinatorial analog of the conjecture fails; indeed, the torsion group of finite permutations of $\mathbb{N}$ is highly transitive. The same holds for the infinite alternating group, that is, the simple group of finite even permutations of $\mathbb{N}$.

Conjecture 1 is inspired in turn by the following question proposed by J.-P. Demailly:

Question 1. What can one say about the growth of the group

$$
G=\left\langle U_{1}, \ldots, U_{s}\right\rangle
$$

generated by a sequence of one-parameter unipotent subgroups, meaning by "growth" the maximal growth of the finitely generated subgroups of $G$ ?

For instance, the group $G$ in Conjecture 1 has exponential growth provided the conjecture is true. Anyway, this group $G$ cannot have a polynomial growth, see Proposition 5.5. The group $G$ in Theorem 1.1 is of polynomial growth in case (i), and of exponential growth in case (ii); the latter holds as well for the group $G$ in Corollary 1.2 , In the combinatorial setup, we do not know the answer to the following general question.

Question 2. Let $G$ be a finitely generated group. Assume $G$ acts highly transitively on a set X. Can $G$ be of intermediate growth?

See, e.g., Fima et al., 2015, 2020, Garion et al., 2013, Hull et al., 2016] for recent studies on highly transitive actions of countable groups, and [Hull et al., 2016, Fima et al., 2020] for surveys. However, the groups of algebrogeometric nature that we study in this paper are quite different.

The proof of our main Theorem 1.1 exploits a constructive criterion/algorithm to decide whether the group $G$ in this theorem is a unipotent algebraic group. We introduce a combinatorial data associated to the given collection of the one-parameter unipotent subgroups $U_{1}, \ldots, U_{s}$ acting on our toric variety $X$. This data is expressed in terms of Demazure roots $(\rho, e)$. To a Demazure root there corresponds a root derivation $\partial_{\rho, e}$ acting on the structure ring $\mathcal{O}(X)$. It can be viewed as a vector field, and it generates a root subgroup $U_{\rho, e}$. If $G$ does not contain any non-abelian free group, then there are strong constraints on the Lie brackets between the root derivations generating the root subgroups of $G$; namely, the bracket of any two such derivations is proportional to one of them. These constraints are encoded in a directed graph $\Gamma$ whose vertices are certain abelian Lie algebras which are indexed via the facets of the associated polyhedral cone of $X$ and generated by the corresponding root derivations; see Definition 4.5. Any edge of $\Gamma$ is oriented in the direction of the bracket of its end vertices provided the corresponding subalgebras do not commute; otherwise, the edge is absent. The geometry of $\Gamma$ determines the structure of $G$. It occurs that $\Gamma$ has no oriented cycle if and only if it has no bidirected edge, if and only if $G$ is a unipotent algebraic group, see Proposition 4.8. Theorem 1.1 is a byproduct of this criterion.

The content of this paper is as follows. Besides the Introduction, the paper includes four sections. Section 2 contains the notation and preliminary facts from toric geometry. In Section 3 we prove Theorem 1.1 in the particular case of a group $G$ generated by just two root subgroups, see Proposition 3.1 The main results of subsections 4.1 and 4.2 are Propositions 4.7 and 4.8, respectively. The former contains a combinatorial criterion for a Lie algebra of derivations to be nilpotent and finite dimensional. The latter provides, in our framework, a link between nilpotent Lie algebras and unipotent algebraic groups. Together, these give Proposition 4.1 which says essentially that if any two root subgroups of the group $G=\left\langle U_{1}, \ldots, U_{s}\right\rangle$ generate a unipotent algebraic group, then $G$ itself is a unipotent algebraic group. Theorem 1.1 follows immediately from Propositions 3.1 and 4.1 . Corollary 1.2 follows from this theorem due to Proposition 5.2 in subsection 5.1. According to this proposition, a unipotent linear algebraic group cannot act 2-transitively on an algebraic variety. Finally, in subsection 5.2 we establish that a virtually solvable group cannot be highly transitive, or even be a subnormal subgroup of a highly transitive group; see Proposition 5.5. In particular, a highly transitive group is of exponential growth provided it satisfies the Tits' type alternative; cf. Question 2.

\section{Preliminaries from toric geometry}

We start by recalling the standard notation and definitions of toric geometry.

\subsection{Toric affine varieties}

Consider an algebraic torus $\mathbb{T}=\left(\mathbb{G}_{m}\right)^{n}$. Let $N$ be the lattice of one-parameter subgroups of $\mathbb{T}, N^{\vee}=$ $\operatorname{Hom}\left(\mathbb{T}, \mathbb{G}_{m}\right)$ the dual lattice of characters, and $\langle\cdot, \cdot\rangle: N \times N^{\vee} \rightarrow \mathbb{Z}$ the natural pairing; see, e.g., Cox et al., 
2011] or [6, Def. 4.2]. Let $\chi^{m}$ stand for the character of $\mathbb{T}$ which corresponds to $m \in N^{\vee}$, so that $\chi^{m} \chi^{m^{\prime}}=\chi^{m+m^{\prime}}$. The group algebra $\mathbf{k}\left[N^{\vee}\right]=\oplus_{m \in N^{\vee}} \mathbf{k} \chi^{m}$ can be identified with the structure algebra $\mathcal{O}(\mathbb{T})$.

Consider further the pair of dual $\mathbb{Q}$-vector spaces $N_{\mathbb{Q}}=N \otimes \mathbb{Q}$ and $N_{\mathbb{Q}}^{\vee}=N^{\vee} \otimes \mathbb{Q}$, a closed polyhedral cone $\Delta_{\mathbb{Q}} \subset N_{\mathbb{Q}}$ and its dual cone $\Delta_{\mathbb{Q}}^{\vee} \subset N_{\mathbb{Q}}^{\vee}$. By abuse of language, by the associated pair of lattice cones we mean the pair $\left(\Delta, \Delta^{\vee}\right)$, where $\Delta=N \cap \Delta_{\mathbb{Q}}$ and $\Delta^{\vee}=N^{\vee} \cap \Delta_{\mathbb{Q}}^{\vee}$, respectively. With any (closed) polyhedral lattice cone $\Delta \subset N$ one associates the normal toric affine variety

$$
X=\operatorname{Spec}\left(\bigoplus_{m \in \Delta^{\vee}} \mathbf{k} \chi^{m}\right)
$$

and any normal toric affine variety arises in this way. The $\mathbb{T}$-action on $X$ is induced by the $\Delta^{\vee}$-grading on the structure algebra $\mathcal{O}(X)$. By Gordon's Lemma Cox et al., 2011, Prop. 1.2.17], the cones $\Delta$ and $\Delta^{\vee}$ are both finitely generated monoids. The lattice vectors $\left(\rho_{j}\right)_{j=1, \ldots, k}$ on the extremal rays of $\Delta_{\mathbb{Q}}$, which are elements of the minimal system of generators of $\Delta$, are called ray generators. These are in one-to-one correspondence with the facets of the dual polyhedral cone $\Delta_{\mathbb{Q}}^{\vee}$. The variety $X$ has no torus factor if and only if $\Delta_{\mathbb{O}}$ is full dimensional, if and only if $\Delta_{\mathbb{Q}}^{\vee} \subset N_{\mathbb{Q}}^{\vee}$ is pointed, that is, contains no affine line. See also Cox et al., 2011, Fulton, 1993, Oda, 1988] for a detailed exposition.

\subsection{Root derivations and root subgroups}

The Demazure facets

$$
S_{j}=\left\{e \in N^{\vee} \mid\left\langle\rho_{j}, e\right\rangle=-1, \quad\left\langle\rho_{i}, e\right\rangle \geq 0, \quad i=1, \ldots, k, \quad i \neq j\right\}, \quad j=1, \ldots, k
$$

are lattice polyhedra in $N^{\vee}$. Each of them is contained in a hyperplane of $N_{\mathbb{Q}}^{\vee}$ parallel to a facet of $\Delta_{\mathbb{Q}}^{\vee}$ and situated outside the cone $\Delta_{\mathbb{Q}}^{\vee}$. The lattice vectors $e \in \bigcup_{j=1}^{k} S_{j}$ are called Demazure roots. Any Demazure facet contains an infinite set of Demazure roots. To a Demazure root $e \in S_{j}$ one associates the root derivation $\partial_{\rho_{j}, e} \in \operatorname{Der}(\mathcal{O}(X))$, which acts on the character $\chi^{m}$ via

$$
\partial_{\rho_{j}, e}\left(\chi^{m}\right)=\left\langle\rho_{j}, m\right\rangle \chi^{m+e}
$$

The kernel of $\partial_{\rho_{j}, e}$ is spanned by the characters $\chi^{m}$, where $m$ runs over the facet of $\Delta^{\vee}$ defined by $\left\langle\rho_{j}, m\right\rangle=0$.

The root derivations are precisely the homogeneous locally nilpotent derivations of the graded algebra $\mathcal{O}(X)=\bigoplus_{m \in \Delta^{\vee}} \mathbf{k} \chi^{m}$. Recall [Liendo, 2010, Thm. 2.4] that any homogeneous derivation of $\mathcal{O}(X)$ is proportional to one of the form $\partial_{\rho, e}$ for some $\rho \in N$ and $e \in N^{\vee}$ acting via

$$
\partial_{\rho, e}\left(\chi^{m}\right)=\langle\rho, m\rangle \chi^{m+e}
$$

where $e$ is called the degree of $\partial_{\rho, e}$. One has [Romaskevich, 2014, Sect. 3]

$$
\left[\partial_{1}, \partial_{2}\right]=\partial_{\rho, e_{1}+e_{2}} \quad \text { with } \quad \rho=d \rho_{2}-c \rho_{1}
$$

The root subgroups $\exp \left(t \partial_{\rho_{j}}, e\right)$ are precisely the $\mathbb{G}_{a}$-subgroups of $\operatorname{Aut}(X)$ normalized by the torus $\mathbb{T}$. See, e.g., Arzhantsev et al., 2019, 2021, Freudenburg, 2017, Liendo, 2010] for further details.

\subsection{Cox rings and total coordinates}

Let $X$ be a normal toric affine variety $X$ with no torus factor. The divisor class group $\mathrm{Cl}(X)$ is the abelian group generated by the classes of the prime $\mathbb{T}$-invariant divisors $D_{1}, \ldots, D_{k}$ on $X$. These divisors are in one-to-one correspondence with the ray generators $\left(\rho_{j}\right)_{j=1, \ldots, k}$. The Cox ring of $X$ is the polynomial ring $\mathcal{O}\left(\mathbb{A}^{k}\right)=\mathbf{k}\left[x_{1}, \ldots, x_{k}\right]$ on a distinguished set of variables called the total coordinates. It is equipped with a $\mathrm{Cl}(X)$-grading defined by $\operatorname{deg}\left(x_{i}\right)=\left[D_{i}\right], i=1, \ldots, k$. This grading corresponds to a diagonal action on $\mathbb{A}^{k}=\operatorname{Spec}\left(\mathbf{k}\left[x_{1}, \ldots, x_{k}\right]\right)$ of the Cox quasitorus $F_{\mathrm{Cox}}=\operatorname{Hom}\left(\mathrm{Cl}(X), \mathbb{G}_{m}\right)$. Recall that a quasitorus is a direct product of an algebraic torus and a finite abelian group. One has [Arzhantsev et al., 2015, Thm. 2.1.3.2]

$$
X \cong \operatorname{Spec}\left(\mathcal{O}\left(\mathbb{A}^{k}\right)^{F_{\text {Cox }}}\right)=\mathbb{A}^{k} / / F_{\text {Cox }}
$$

See also Arzhantsev et al., 2015, 2019], Cox et al., 2011, Ch. 5].

Lemma 2.1. Let $e \in S_{j}$ be a Demazure root, and let $\hat{e}=\left(c_{1}, \ldots, c_{k}\right) \in \mathbb{Z}^{k}$, where $c_{i}=\left\langle\rho_{i}, e\right\rangle$. Then the following hold. 
(a) The integer lattice vector $\hat{e}$ is a Demazure root of $\mathbb{A}^{k}$ (viewed as a toric variety with the standard action of the $k$-torus) which belongs to the $j$ th Demazure facet $\hat{S}_{j}$ of the first octant $\mathbb{Z}_{\geq 0}^{k} \subset \mathbb{Z}^{k}$.

(b) Let $\left(\varepsilon_{i}\right)_{i=1, \ldots, k}$ be the ray generators of the lattice cone $\mathbb{Z}_{\geq 0}^{k}$. Then one has

$$
\hat{\partial}:=\partial_{\varepsilon_{j}, \hat{e}}=M_{j} \frac{\partial}{\partial x_{j}}, \quad \text { where } \quad M_{j}=x_{1}^{c_{1}} \cdots x_{j-1}^{c_{j-1}} x_{j+1}^{c_{j+1}} \cdots x_{k}^{c_{k}} \in \mathbf{k}\left[x_{1}, \ldots, x_{j-1}, x_{j+1}, \ldots, x_{k}\right] .
$$

The associated $\mathbb{G}_{a}$-subgroup consists of elementary transformations

$$
\exp (t \hat{\partial}):\left(x_{1}, \ldots, x_{k}\right) \mapsto\left(x_{1}, \ldots, x_{j-1}, x_{j}+t M_{j}, x_{j+1}, \ldots, x_{k}\right), \quad t \in \mathbf{k}
$$

This is a subgroup of the tame automorphism group Tame $\left(\mathbb{A}^{k}\right)$.

(c) The Cox quasitorus $F_{\text {Cox }}$ and the $\mathbb{G}_{a}$-subgroup $\exp (t \hat{\partial})$ commute in $\operatorname{Aut}\left(\mathcal{O}\left(\mathbb{A}^{k}\right)\right)$, and

$$
\left.\exp (t \hat{\partial})\right|_{\mathcal{O}\left(\mathbb{A}^{k}\right)^{F_{\text {Cox }}}}=\exp \left(t \partial_{\rho_{j}, e}\right)
$$

(d) Given a sequence $\left(\partial_{1}, \ldots, \partial_{s}\right)$ of root derivations of $\mathcal{O}(X)$, where $\partial_{i}=\partial_{\rho_{j(i)}, e_{i}}$ with a Demazure root $e_{i} \in S_{j(i)}$ of $X$, and the sequence of the corresponding root derivations $\hat{\partial}_{i}=\partial_{\varepsilon_{j(i)}, \hat{e}_{i}}$ of the $\operatorname{Cox} \operatorname{ring} \mathcal{O}\left(\mathbb{A}^{k}\right)=$ $\mathbf{k}\left[x_{1}, \ldots, x_{k}\right]$ with $\hat{e}_{i} \in \hat{S}_{j(i)}, i=1, \ldots, s$, consider the Lie algebras $L$ and $\hat{L}$ generated, respectively, by $\partial_{1}, \ldots, \partial_{s}$ and $\hat{\partial}_{1}, \ldots, \hat{\partial}_{s}$. Then the correspondence $\partial_{i} \mapsto \hat{\partial}_{i}, i=1, \ldots, s$, induces an isomorphism of Lie algebras $L \cong \hat{L}$.

Proof. Statement (a) is immediate; statements (b) and (c) follow easily from Arzhantsev et al., 2019, Lem. 4.20.b]; see Arzhantsev et al., 2019, (12)] for (2). To show (d), consider the morphism $\pi: \mathbb{A}^{k} \rightarrow X=$ $\mathbb{A}^{k} / / F_{\text {Cox }}$. The induced pullback homomorphism $\pi^{*}: \mathcal{O}(X) \rightarrow \mathcal{O}\left(\mathbb{A}^{k}\right)$ is injective, and its image coincides with the algebra of invariants $\mathcal{O}\left(\mathbb{A}^{k}\right)^{F_{\text {Cox }}}$. The induced homomorphism of the Lie algebras of vector fields $\pi^{*}: \operatorname{Vec}(X) \rightarrow \operatorname{Vec}\left(\mathbb{A}^{k}\right)$ is as well injective, and its image coincides with the Lie subalgebra of $F_{\text {Cox }}$-invariant vector fields on $\mathbb{A}^{k}$ yielding an isomorphism $\operatorname{Vec}(X) \cong \operatorname{Vec}\left(\mathbb{A}^{k}\right)^{F_{\text {Cox }}}$. Considering the derivations as vector fields, we have $\pi^{*}\left(\partial_{i}\right)=\hat{\partial}_{i}, i=1, \ldots, s$, and $\pi^{*}(L)=\hat{L}$.

Recall that a linear algebraic group is called unipotent if it consists of unipotent matrices. In characteristic zero, any unipotent algebraic group is isomorphic to an affine space $\mathbb{A}^{n}$ as a variety. Any orbit of a unipotent algebraic group acting regularly on an affine variety is closed and isomorphic to an affine space. In the sequel we need the following technical results.

Proposition 2.2. Given a collection of Demazure roots $\left(e_{j(i), i} \in S_{j(i)}\right)_{i=1, \ldots, s}$, let

$$
G=\left\langle U_{i} \mid i=1, \ldots, s\right\rangle \subset \operatorname{Aut}(X) \quad \text { where } \quad U_{i}=\exp \left(t \partial_{\rho_{j(i)}, e_{j(i), i}}\right) .
$$

Consider the root derivations $\hat{\partial}_{i}=\hat{\partial}_{\varepsilon_{j(i)}, \hat{e}_{j(i), i}}$ and the root subgroups $\hat{U}_{i}=\exp \left(t \hat{\partial}_{i}\right) \operatorname{acting}$ on $\mathbb{A}^{k}, i=1, \ldots, s$. Let

$$
\hat{G}=\left\langle\hat{U}_{i} \mid i=1, \ldots, s\right\rangle \subset \operatorname{Aut}\left(\mathbb{A}^{k}\right) .
$$

Then the following holds.

(a) If $\hat{G}$ contains a free subgroup $\mathbf{F}_{m}$ of rank $m \geq 2$ then $G$ does.

(b) If $\hat{G}$ is a unipotent algebraic group then $G$ is, and, moreover, $G \cong \hat{G}$.

Proof. (a) Since any subgroup $\hat{U}_{i}, i=1, \ldots, s$ commutes with the quasitorus $F_{\text {Cox }}$ in $\operatorname{Aut}\left(\mathbb{A}^{k}\right)$ one has

$$
\hat{G} \subset \operatorname{Centr}_{\mathrm{Aut}\left(\mathbb{A}^{k}\right)}\left(F_{\mathrm{Cox}}\right) \subset \operatorname{Norm}_{\mathrm{Aut}\left(\mathbb{A}^{k}\right)}\left(F_{\mathrm{Cox}}\right),
$$

where $\operatorname{Centr}_{\operatorname{Aut}\left(\mathbb{A}^{k}\right)}\left(F_{\text {Cox }}\right)$ and $\operatorname{Norm}_{\operatorname{Aut}\left(\mathbb{A}^{k}\right)}\left(F_{\text {Cox }}\right)$ are the centralizer and the normalizer of $F_{\text {Cox }}$ in $\operatorname{Aut}\left(\mathbb{A}^{k}\right)$, respectively. There is the exact sequence [Arzhantsev et al., 2010, Thm. 5.1]

$$
1 \rightarrow F_{\mathrm{Cox}} \rightarrow \operatorname{Norm}_{\mathrm{Aut}\left(\mathbb{A}^{k}\right)}\left(F_{\mathrm{Cox}}\right) \stackrel{\tau}{\longrightarrow} \operatorname{Aut}(X) \rightarrow 1 .
$$


Assume $\hat{G}$ contains a free subgroup $\mathbf{F}_{m}$ of rank $m \geq 2$. We claim that the restriction

$$
\left.\tau\right|_{\mathbf{F}_{m}}: \mathbf{F}_{m} \rightarrow \mathbf{F}_{m} /\left(\mathbf{F}_{m} \cap F_{\text {Cox }}\right) \subset \operatorname{Aut}(X)
$$

is an isomorphism, that is, $\mathbf{F}_{m} \cap F_{\text {Cox }}=1$. Indeed, the latter intersection is a normal abelian subgroup of the non-abelian free group $\mathbf{F}_{m}$, hence the trivial group.

(b) Suppose $\hat{G}$ is a unipotent algebraic group. Then, once again, the restriction

$$
\left.\tau\right|_{\hat{G}}: \hat{G} \rightarrow \hat{G} /\left(\hat{G} \cap F_{\text {Cox }}\right) \subset \operatorname{Aut}(X)
$$

is an isomorphism, that is, $\hat{G} \cap F_{\text {Cox }}=1$. Indeed, the unipotent linear algebraic group $\hat{G}$ has no torsion. Hence, $\hat{G} \cap F_{\text {Cox }}$ is an algebraic subgroup of the quasitorus $F_{\text {Cox }}$. Since this subgroup has no torsion, this is the trivial group.

Remark 2.3. In the proof we have used the fact that $\mathbf{F}_{m}, m>1$, does not contain any normal abelian subgroup. Following suggestions of a referee, we indicate two alternative arguments (our initial proof was more complicated). A simple direct argument is as follows. Let $A$ be a nontrivial abelian normal subgroup of the free group $\mathbf{F}_{m}=\left\langle u_{1}, \ldots, u_{m}\right\rangle$. Then $A$ is cyclic, say $A=a^{\mathbb{Z}}$; indeed, any subgroup of $\mathbf{F}_{m}$ is free. Up to conjugacy, we may assume that $a$ is cyclically reduced, starting with letter $u_{1}$ and finishing with letter $u_{m}$, say. The group $\mathbf{F}_{m}$ acts on $A$ via conjugation, and any automorphism of $A$ sends its generator $a$ to $a^{ \pm 1}$. So, $w a w^{-1}=a^{ \pm 1}$ for any $w \in \mathbf{F}_{m}$. However, the length of $u_{1} a u_{1}^{-1}$ differs from the length of $a^{ \pm 1}$, a contradiction.

Alternatively, there is a nice geometric argument. The natural action of $A$ fixes two points on the boundary $\partial \mathbf{F}_{m}$, namely, the ends of the Caley graph of $A \cong \mathbb{Z}$. Since $A$ is normal, these two points form an invariant set of $\partial \mathbf{F}_{m}$. However, no finite set is fixed by the $\mathbf{F}_{m}$-action on $\partial \mathbf{F}_{m}$.

More generally, no nontrivial abelian subgroup of $\mathbf{F}_{m}, m>1$, is subnormal (see subsection 5.2 for the definition). Indeed, assume there is a descending series $\mathbf{F}_{m} \unrhd N_{1} \unrhd \ldots \unrhd N_{s} \unrhd A$, where $A \neq 1$ is abelian, hence a free cyclic group. One may suppose that $N_{s}$ is a non-abelian free group of finite rank, and then the previous result applied to the pair $\left(N_{s}, A\right)$ gives a contradiction.

\section{Tits' type alternative for a pair of root subgroups}

In this section we still deal with a toric affine variety $X$ over $\mathbf{k}$ with no torus factor, and freely use the notation from 2.1 2.3. We prove the following partial result; cf. Theorem 1.1 .

Proposition 3.1. Consider the group $H=\left\langle U_{1}, U_{2}\right\rangle \subset \operatorname{Aut}(X)$ generated by the root subgroups $U_{i}=\exp \left(t \partial_{i}\right)$, $i=1,2$, associated with two different ray generators, say, $\rho_{1}$ and $\rho_{2}$, respectively. Then either $H$ is a unipotent algebraic group, or $H$ contains a free subgroup of rank 2 .

Proof. Introducing the total coordinates $\left(x_{1}, \ldots, x_{k}\right)$, we let $U_{1}$ and $U_{2}$ act on $\mathbb{A}^{k}$ as $\mathbb{G}_{a}$-subgroups $\hat{U}_{1}$ and $\hat{U}_{2}$ of the tame automorphism group Tame $\left(\mathbb{A}^{k}\right)$ commuting with the Cox quasitorus $F_{\text {Cox }}$, see Lemma 2.1. We let $\hat{H}=\left\langle\hat{U}_{1}, \hat{U}_{2}\right\rangle$. By Proposition 2.2 it suffices to prove the above alternative for $\hat{H}$ instead of $H$.

Let in these coordinates $\hat{e}_{i}=\left(c_{i j}\right)$ where $c_{i i}=-1$ and $c_{i j} \geq 0$ for $j \neq i, i \in\{1,2\}$. One can write

$$
\hat{e}_{1}=(-1, c, *, \ldots, *) \quad \text { and } \quad \hat{e}_{2}=(d,-1, *, \ldots, *), \quad \text { where } c=\left\langle\rho_{2}, e_{1}\right\rangle, \quad d=\left\langle\rho_{1}, e_{2}\right\rangle,
$$

and the stars stand for nonnegative integers. The elements $\hat{u}_{i} \in \hat{U}_{i}, i=1,2$ can be written as

$$
\hat{u}_{1}=\left(x_{1}+s x_{2}^{c} N_{1}, x_{2}, \ldots, x_{k}\right) \text { and } \hat{u}_{2}=\left(x_{1}, x_{2}+t x_{1}^{d} N_{2}, x_{3}, \ldots, x_{k}\right),
$$

where $s, t \in \mathbf{k}$ and $N_{1}, N_{2} \in \mathbf{k}\left[x_{3}, \ldots, x_{k}\right]$ are nonzero monomials, cf. (2)-(3).

By (11), $\hat{H}$ is abelian (and then $\hat{H} \cong \mathbb{G}_{a} \times \mathbb{G}_{a}$ ) if and only if $c=d=0$. More generally, the following holds.

Claim 1. Assume $c>0$ and $d=0$. Then $\hat{H}=\left\langle\hat{U}_{1}, \hat{U}_{2}\right\rangle$ is a unipotent linear algebraic group.

Proof of Claim 1. Under our assumptions, $\hat{H}$ is a closed subgroup of the unipotent linear algebraic group consisting of the triangular transformations

$$
\left(x_{1}, \ldots, x_{k}\right) \mapsto\left(x_{1}+F\left(x_{2}, N_{2}\right) N_{1}, x_{2}+t N_{2}, x_{3}, \ldots, x_{k}\right),
$$

where $t \in \mathbf{k}$ and $F$ runs over the linear space of homogeneous polynomials in two variables of degree $c$. So, $\hat{H}$ is a unipotent linear algebraic group. 固

¥Alternatively, one can deduce the conclusion by using Proposition 4.8 
Suppose now that $c \geq 1$ and $d \geq 1$. In this case we show, using ping-pong type arguments, that $\hat{H}$ contains a free subgroup of rank two, see Claims $2-4$. We analyze separately the cases $c, d \geq 2, c \geq 2$ and $d=1$, and $c=d=1$. This analysis is close to the original Jung approach in [Jung, 1942]; cf. also [Kambavashi, 1979, Lem. 4.1] and [Wright, 1975, 5.31, p. 65]. Another reference in order is [Lamy, 2001], where the enhanced Tits alternative for the group $\operatorname{Aut}\left(\mathbb{A}_{\mathbb{C}}^{2}\right)$ was established playing the ping-pong on the Bass-Serre tree. On can apply this alternative to the group $\operatorname{Aut}\left(\mathbb{A}_{K}^{2}\right)$, where $K$ is the rational function field $\mathbf{k}\left(x_{3}, \ldots, x_{n}\right)$.

Notice that by (6), any $\hat{h} \in \hat{H}$ can be written as

$$
\hat{h}=\left(p, q, x_{3}, \ldots, x_{k}\right) \quad \text { with } \quad p, q \in \mathbf{k}\left[x_{1}, \ldots, x_{k}\right] \backslash \mathbf{k} .
$$

Claim 2. Assume $c, d \geq 2$. Then one has $\hat{H}=\hat{U}_{1} * \hat{U}_{2} \cong \mathbb{G}_{a} * \mathbb{G}_{a}$. Consequently, any two non-unit elements $\hat{u}_{i} \in \hat{U}_{i}, i=1,2$, generate a free subgroup of rank two.

Proof of Claim 2. Fixing $\hat{u}_{i} \in \hat{U}_{i}, i=1,2$ as in (6) with nonzero $s, t \in \mathbf{k}$, for $\hat{h}$ as in (7) one has

$$
\hat{u}_{1} \hat{h}=\left(p_{1}, q, x_{3}, \ldots, x_{k}\right) \text { and } \hat{u}_{2} \hat{h}=\left(p, q_{2}, x_{3}, \ldots, x_{k}\right),
$$

where by (6),

For $\operatorname{deg}(p) \leq \operatorname{deg}(q)$ one gets

$$
p_{1}=p+s q^{c} N_{1} \quad \text { and } \quad q_{2}=q+t p^{d} N_{2}
$$

and, similarly, for $\operatorname{deg}(p) \geq \operatorname{deg}(q)$ one deduces

$$
\operatorname{deg}\left(p_{1}\right)=c \operatorname{deg}(q)+\operatorname{deg}\left(N_{1}\right)>\operatorname{deg}(q),
$$

$$
\operatorname{deg}\left(q_{2}\right)>\operatorname{deg}(p)
$$

Consider a nontrivial reduced word $w$ in two letters, and let $\hat{h}=w\left(\hat{u}_{1}, \hat{u}_{2}\right) \in \hat{H}$, where $\hat{u}_{1}, \hat{u}_{2} \neq 1$. Using (9) -(10) one concludes by recursion on the length of $w$ that $\operatorname{deg}(p)>\operatorname{deg}(q)$ if $w\left(\hat{u}_{1}, \hat{u}_{2}\right)$ starts on the left with $\hat{u}_{1}$, and $\operatorname{deg}(p)<\operatorname{deg}(q)$ if $w\left(\hat{u}_{1}, \hat{u}_{2}\right)$ starts with $\hat{u}_{2}$. Anyway, $\operatorname{deg}(p) \neq \operatorname{deg}(q)$, hence $\hat{h} \neq 1$.

Claim 3. The conclusion of Claim 2 remains valid if $c \geq 2$ and $d=1$.

Proof of Claim 3. For any field $F$ the Jung-van der Kulk Theorem [Jung, 1942, van der Kulk, 1953] yields the presentation

$$
\operatorname{Aut}\left(\mathbb{A}_{F}^{2}\right)=A *_{C} J
$$

where $C=A \cap J, A=\operatorname{Aff}\left(\mathbb{A}_{F}^{2}\right)$ is the affine group of the plane, and $J$ is the de Jonquières $\operatorname{subgroup~of~} \operatorname{Aut}\left(\mathbb{A}_{F}^{2}\right)$ which consists of the transformations of the form

$$
\left(x_{1}, x_{2}\right) \mapsto\left(\alpha_{1} x_{1}+\beta_{1}\left(x_{2}\right), \alpha_{2} x_{2}+\beta_{2}\right) \quad \text { with } \quad \alpha_{i} \in F^{*}, i=1,2, \beta_{1} \in F\left[x_{2}\right], \beta_{2} \in F ;
$$

see [Dicks, 1983, Nagata, 1972, Wright, 1975], Kambayashi, 1975, Thm. 2], and Kambavashi, 1979, Lem. 4.1]. Let $\hat{u}_{1}=\hat{u}_{1}(s), \hat{u}_{2}=\hat{u}_{2}(t)$, and $N_{1}, N_{2} \in \mathbf{k}\left[x_{3}, \ldots, x_{k}\right]$ be as in (6). Letting $F=\mathbf{k}\left(x_{3}, \ldots, x_{k}\right)$ and

$$
b_{1}(s)=s N_{1} \in F[s], \quad b_{2}(t)=t N_{2} \in F[t], \quad \hat{u}_{1}=\hat{u}_{1}(s), \quad \hat{u}_{2}=\hat{u}_{2}(t)
$$

one gets

$$
\hat{H}=\left\langle\hat{u}_{1}, \hat{u}_{2}\right\rangle=\left\langle\left(x_{1}+b_{1}(s) x_{2}^{c}, x_{2}\right),\left(x_{1}, x_{2}+b_{2}(t) x_{1}\right) \mid s, t \in \mathbf{k}\right\rangle \subset \operatorname{Aut}\left(\mathbb{A}_{F}^{2}\right),
$$

where $b_{1}(s), b_{2}(t)$ do not vanish for any $(s, t) \in\left(\mathbb{A}^{1} \backslash\{0\}\right)^{2}$. Since $c>1$ and $d=1$, one has

$$
\hat{U}_{1} \backslash\{\mathrm{id}\} \subset \mathrm{J} \backslash \mathrm{C} \text { and } \hat{\mathrm{U}}_{2} \backslash\{\mathrm{id}\} \subset \mathrm{A} \backslash \mathrm{C} \text {. }
$$

Due to (11) any nonunit element $\hat{h} \in \hat{H}$ can be uniquely written as a nonempty alternating product of the type

$$
\hat{h}=\hat{u}_{1}\left(s_{1}\right) \hat{u}_{2}\left(t_{1}\right) \cdots \hat{u}_{1}\left(s_{m}\right) \hat{u}_{2}\left(t_{m}\right),
$$

where $s_{i}, t_{i} \neq 0$, up to omitting the first or the last factor, or the both. Now the claim follows.

The next claim ends the proof of Proposition 3.1.

Claim 4. Assume $c=d=1$. Then there exist $\left(\hat{u}_{1}, \hat{u}_{2}\right) \in \hat{U}_{1} \times \hat{U}_{2}$ such that the group $\left\langle\hat{u}_{1}, \hat{u}_{2}\right\rangle$ surjects onto $\mathrm{SL}_{2}(\mathbb{Z})$ and so, contains a free subgroup of rank two.

Proof of Claim 4. Choosing in (12) the values of parameters $s_{0}, t_{0}$ such that $b_{1}\left(s_{0}\right)=b_{2}\left(t_{0}\right)=1$, by (13) we obtain

$$
\left\langle\hat{u}_{1}\left(s_{0}\right), \hat{u}_{2}\left(t_{0}\right)\right\rangle=\left\langle\left(x_{1}+x_{2}, x_{2}\right),\left(x_{1}, x_{2}+x_{1}\right)\right\rangle=\mathrm{SL}_{2}(\mathbb{Z}) .
$$

This yields the desired surjection $\left\langle\hat{u}_{1}, \hat{u}_{2}\right\rangle \rightarrow \mathrm{SL}_{2}(\mathbb{Z})$. It remains to recall Wikipedia, 3.1] that $\mathrm{SL}_{2}(\mathbb{Z})$ is virtually free with $\left\langle\left(x_{1}+2 x_{2}, x_{2}\right),\left(x_{1}, x_{2}+2 x_{1}\right)\right\rangle \cong \mathbf{F}_{2}$. 
Remark 3.2. By the Kurosh subgroup theorem, under the assumptions of Claims 2 and 3 any nontrivial algebraic subgroup of the free product $\hat{H}=\hat{U}_{1} * \hat{U}_{2}$ is conjugated to one of the factors $\hat{U}_{i}, i=1,2$. Hence, it is isomorphic to $\mathbb{G}_{a}$. By Proposition $2.2(\mathrm{~b})$ the latter remains valid after taking off the hats.

Corollary 3.3. In the notation as before, the following conditions are equivalent:

(i) $\hat{H}=\left\langle\hat{U}_{1}, \hat{U}_{2}\right\rangle$ is a unipotent algebraic group;

(ii) letting $K=\mathbf{k}\left[x_{3}, \ldots, x_{k}\right]$ the Lie algebra $\hat{L}=\operatorname{Lie}\left(\hat{\partial}_{1}, \hat{\partial}_{2}\right) \subset \operatorname{Der}_{K}\left(K\left[x_{1}, x_{2}\right]\right)$ generated by the root derivations (see (6) )

$$
\hat{\partial}_{1}=x_{2}^{c} N_{1} \partial / \partial x_{1}, \quad \hat{\partial}_{2}=x_{1}^{d} N_{2} \partial / \partial x_{2}
$$

is finite dimensional and nilpotent;

$$
\min \left\{\left\langle\hat{\rho}_{1}, \hat{e}_{2}\right\rangle,\left\langle\hat{\rho}_{2}, \hat{e}_{1}\right\rangle\right\}=\min \{c, d\}=0 .
$$

These equivalences remain valid after taking off the hats.

Proof. The implication (i) $\Rightarrow$ (ii) is immediate; indeed, $\hat{L}=\operatorname{Lie}(\hat{H})$ provided (i) is fulfilled. The equivalence (i) $\Leftrightarrow$ (iii) is established in the course of the proof of Proposition 3.1. Hence, it suffices to show (ii) $\Rightarrow$ (iii). Notice that the specialization $\left(x_{1}, \ldots, x_{k}\right) \mapsto\left(x_{1}, x_{2}, x_{3}^{(0)}, \ldots, x_{k}^{(0)}\right)$ yields a surjective homomorphism of Lie algebras $\operatorname{Der}_{K}\left(K\left[x_{1}, x_{2}\right]\right) \rightarrow \operatorname{Der}_{\mathbf{k}}\left(\mathbf{k}\left[x_{1}, x_{2}\right]\right)$. Choosing a point $P_{0}=\left(x_{3}^{(0)}, \ldots, x_{k}^{(0)}\right) \in\left(\mathbb{A}^{1} \backslash\{0\}\right)^{k-2}$ so that $N_{1}\left(P_{0}\right)$ and $N_{2}\left(P_{0}\right)$ do not vanish we may assume that $\hat{\partial}_{1}=y^{c} \partial / \partial x, \hat{\partial}_{2}=x^{d} \partial / \partial y$, and $\hat{L}=\operatorname{Lie}\left(\hat{\partial}_{1}, \hat{\partial}_{2}\right) \subset \operatorname{Der}_{\mathbf{k}}(\mathbf{k}[x, y])$ is finite dimensional and nilpotent. Suppose (iii) fails. If $c=d=1$ then $\hat{L}=\mathrm{sl}_{2}(\mathbf{k})$ is not nilpotent. If, say, $c \geq 1$ and $d>1$ then we have

$$
\operatorname{ad}\left(\hat{\partial}_{1}\right)^{d+1}\left(\hat{\partial}_{2}\right)=-c(d+1) ! y^{e} \partial / \partial x \in \hat{L}, \quad \text { where } \quad e=(d+1) c-1>c .
$$

Replacing now $\hat{\partial}_{1}=y^{c} \partial / \partial x$ by $y^{e} \partial / \partial x$ and repeating the trick, we obtain a sequence of elements of $\hat{L}$ of unbounded degrees. Thus, in this case $\hat{L}$ has infinite dimension. In any case, (ii) fails, a contradiction.

For the last assertion, see Lemma 2.1, d and Proposition 2.2. b.

\section{Tits' type alternative for a sequence of root subgroups}

Let as before $X$ be a toric affine variety with no torus factor, and let

$$
G=\left\langle U_{1}, \ldots, U_{s}\right\rangle
$$

be the group generated by a finite set of root subgroups $U_{j}=\exp \left(t \partial_{j}\right) \subset \operatorname{Aut}(X), j=1, \ldots, s$, where $\partial_{j}$ are root derivations. According to Corollary 3.3, in the case that $G$ does not contain any non-abelian free subgroup, for any $i \neq j$ either $U_{i}$ and $U_{j}$ belong to the same ray generator (and then commute), or they belong to two different ray generators $\rho$ and $\rho^{\prime}$ and for the corresponding roots $e, e^{\prime}$ one has $\min \left\{\left\langle\rho, e^{\prime}\right\rangle,\left\langle\rho^{\prime}, e\right\rangle\right\}=0$. In Proposition 4.1 we establish that under these assumptions $G$ is a unipotent algebraic group. To be more precise, notice that the Lie algebra $L$ generated by the root derivations $\partial_{j}, j=1, \ldots, s$, might contain extra root derivations, cf. Example 4.2. Let $R_{i}$ be the set of Demazure roots $e_{i j} \in S_{i}$ of $X$ such that $\partial_{\rho_{i}, e_{i j}} \in L$. A priori, the cardinal $\operatorname{card}\left(R_{i}\right)$ could be infinite countable, and then the abelian subalgebra

$$
L_{i}=\operatorname{Lie}\left(\partial_{\rho_{i}, e_{i j}} \mid e_{i j} \in R_{i}\right) \subset L
$$

is infinite dimensional. We may suppose that

$$
R_{i} \neq \emptyset \quad \forall i=1, \ldots, r \quad \text { and } \quad R_{i}=\emptyset \quad \forall i=r+1, \ldots, k .
$$

Let $R=\bigcup_{i=1}^{r} R_{i}$. For $e \in R_{i}$ we let $U_{e}=\exp \left(t \partial_{\rho_{i}, e}\right)$.

Proposition 4.1. Suppose that for all $e, e^{\prime} \in R$ the group $\left\langle U_{e}, U_{e^{\prime}}\right\rangle$ is unipotent. Then $G$ is a unipotent algebraic group.

The proof is done at the end of subsection 4.2. The assumption of Proposition 4.1 is equivalent to the fact that $\left\langle U_{e}, U_{e^{\prime}}\right\rangle$ for any $e, e^{\prime} \in R$ does not contain any free subgroup of rank two. The latter is equivalent to the fact that (14) holds for any $e, e^{\prime} \in R$, see Proposition 3.1 and Corollary 3.3. Theorem 1.1 from the introduction is an immediate consequence of Propositions 3.1 and 4.1. In turn, Proposition 4.1 follows from Propositions 4.7 and 4.8 . 


\subsection{Acyclicity and nilpotent Lie algebras}

Before passing to the proof of Proposition 4.1, let us give an example.

Example 4.2. Consider the group $G=\left\langle U_{1}, U_{2}, U_{3}, U_{4}\right\rangle$ acting on $\mathbb{A}^{3}=\operatorname{Spec} \mathbf{k}[x, y, z]$, where $U_{i}=\exp \left(t \partial_{i}\right)$, $i=1, \ldots, 4$ with

$$
\partial_{1}=y z \frac{\partial}{\partial x}, \quad \partial_{2}=z \frac{\partial}{\partial y}, \quad \partial_{3}=z^{2} \frac{\partial}{\partial y}, \quad \partial_{4}=\frac{\partial}{\partial z} .
$$

We have

$$
\partial_{1}=\partial_{\rho_{1}, e_{1}}, \quad \partial_{2}=\partial_{\rho_{2}, e_{2}}, \quad \partial_{3}=\partial_{\rho_{2}, e_{3}}, \quad \partial_{4}=\partial_{\rho_{3}, e_{4}},
$$

where the ray generators $\rho_{1}, \rho_{2}, \rho_{3}$ are the vectors of the standard basis in $\mathbb{A}^{3}$, and

$$
e_{1}=(-1,1,1), \quad e_{2}=(0,-1,1), \quad e_{3}=(0,-1,2), \quad e_{4}=(0,0,-1) .
$$

Any pair of these root derivations verify (14). They generate the Lie algebra

$$
L=\operatorname{span}\left(\frac{\partial}{\partial x}, y \frac{\partial}{\partial x}, y z \frac{\partial}{\partial x}, z \frac{\partial}{\partial x}, z^{2} \frac{\partial}{\partial x}, z^{3} \frac{\partial}{\partial x}, \frac{\partial}{\partial y}, z \frac{\partial}{\partial y}, z^{2} \frac{\partial}{\partial y}, \frac{\partial}{\partial z}\right) .
$$

Consider the abelian Lie subalgebras

$$
\begin{gathered}
L_{1}=\operatorname{span}\left(\frac{\partial}{\partial x}, y \frac{\partial}{\partial x}, y z \frac{\partial}{\partial x}, z \frac{\partial}{\partial x}, z^{2} \frac{\partial}{\partial x}, z^{3} \frac{\partial}{\partial x}\right), \\
L_{2}=\operatorname{span}\left(\frac{\partial}{\partial y}, z \frac{\partial}{\partial y}, z^{2} \frac{\partial}{\partial y}\right), \quad \text { and } \quad L_{3}=\operatorname{span}\left(\frac{\partial}{\partial z}\right) .
\end{gathered}
$$

We have

$$
L=L_{1} \oplus L_{2} \oplus L_{3}, \quad \text { where } \quad\left[L_{1}, L_{i}\right] \subset L_{1}, i=2,3, \quad\left[L_{2}, L_{3}\right] \subset L_{2},
$$

and, furthermore,

$$
\operatorname{ad}\left(L_{i}\right)\left(L_{i}\right)=0, i=1,2,3, \quad \operatorname{ad}\left(L_{3}\right)^{4}\left(L_{1}\right)=0, \quad \operatorname{ad}\left(L_{2}\right)^{2}\left(L_{1}\right)=0, \quad \operatorname{ad}\left(L_{3}\right)^{3}\left(L_{2}\right)=0 .
$$

For the lower central series $L^{i}=\left[L, L^{i-1}\right]$ of $L$ we obtain $L^{5}=0$. Thus, $L$ is nilpotent, and so, by Proposition 4.8, $G$ is a unipotent algebraic group.

The proof of Proposition 4.1 is based on Proposition 4.7, which strengthens Arzhantsev et al., 2021, Thm. 5.1] in our particular context. Let us recall the terminology of [Arzhantsev et al., 2021] and introduce the necessary notation.

Definition 4.3. Consider a finite sequence of root derivations

$$
\mathcal{D}=\left(D_{1}, \ldots, D_{t}, D_{t+1}\right) \text { where } D_{i}=\partial_{\rho_{j(i)}, e_{j(i), i}} \in L_{j(i)} \text { with } e_{j(i), i} \in R_{j(i)}, j(i) \in\{1, \ldots, r\} .
$$

One says that $\mathcal{D}$ is a cycle (more precisely, a $t$-cycle) if $D_{t+1}=D_{1}$ and

$$
\left\langle\rho_{j(i+1)}, e_{j(i), i}\right\rangle>0 \quad \forall i=1, \ldots, t .
$$

For instance, $\left(D_{1}, D_{2}, D_{1}\right)$ forms a 2-cycle if and only if (14) fails, that is,

$$
\left\langle\rho_{j(2)}, e_{j(1), 1}\right\rangle>0 \text { and }\left\langle\rho_{j(1)}, e_{j(2), 2}\right\rangle>0 .
$$

We say that $\mathcal{D}$ is a pseudo-cycle if (16) holds and $j(t+1)=j(1)$, but not necessarily $e_{j(t+1), t+1}=e_{j(1), 1}$; that is, $\rho_{j(t+1)}=\rho_{j(1)}$ but possibly $D_{t+1} \neq D_{1}$.

In this subsection we mainly deal with the case where $G$ contains no non-abelian free subgroup, or, which is equivalent, $L$ contains no 2-cycle of root derivations. We need the next technical lemma.

Lemma 4.4. The following conditions are equivalent:

(i) $L$ contains no 2-cycle of root derivations;

(ii) $L$ contains no 2-pseudo-cycle of root derivations; 
(iii) for any pair of indices $i, j \in\{1, \ldots, r\}$ such that $i \neq j$, at least one of the abelian Lie subalgebras $L_{i}, L_{j}$ from (15) is an ideal of the Lie algebra Lie $\left(L_{i}, L_{j}\right)$;

(iv) the Lie algebra $\operatorname{Lie}\left(\partial_{\rho_{\mathrm{i}}, \mathrm{e}}, \partial_{\rho_{\mathrm{j}}, \mathrm{e}^{\prime}}\right)$ is finite dimensional and nilpotent for any pair of indices $i, j \in\{1, \ldots, r\}$ and any pair of roots $e \in R_{i}, e^{\prime} \in R_{j}$.

Proof. (i) $\Leftrightarrow$ (ii). Assume (i) holds. Then we have

$$
\min \left\{\left\langle\rho_{i}, e^{\prime}\right\rangle,\left\langle\rho_{j}, e\right\rangle\right\}=0 \quad \forall e \in R_{i}, \quad \forall e^{\prime} \in R_{j} \quad \text { with } \quad 1 \leq i \neq j \leq r
$$

Condition (ii) is clearly fulfilled if $L_{i}$ and $L_{j}$ commute. Otherwise, up to interchanging $i$ and $j$, there exists $e_{i} \in R_{i}$ such that $\left\langle\rho_{j}, e_{i}\right\rangle=c>0$, see (11). By virtue of (17) one has

$$
\left\langle\rho_{i}, e^{\prime}\right\rangle=0 \quad \forall e^{\prime} \in R_{j}
$$

It follows that $L$ has no 2-pseudo-cycle, that is, (ii) holds. The converse implication is immediate.

(ii) $\Leftrightarrow$ (iii). Assume (ii) holds. Then (17) is fulfilled. As before, (iii) is evidently true if $L_{i}$ and $L_{j}$ commute. Suppose this is not the case, and let $\left\langle\rho_{j}, e_{i}\right\rangle=c>0$ for some $e_{i} \in R_{i}$. From (11) and (18) one deduces that $e_{i}+e^{\prime} \in R_{i}$ for any $e^{\prime} \in R_{j}$, and

$$
\left[\partial_{\rho_{j}, e^{\prime}}, \partial_{\rho_{i}, e_{i}}\right]=c \partial_{\rho_{i}, e_{i}+e^{\prime}} \in L_{i} \forall e^{\prime} \in R_{j}
$$

that is,

$$
0 \neq\left[L_{j}, L_{i}\right] \subset L_{i} .
$$

Thus, (iii) is fulfilled. To show the converse, notice that $\left[L_{j}, L_{i}\right] \subset L_{i}$ for $i \neq j$ implies (19) for any $e_{i} \in R_{i}, e^{\prime} \in R_{j}$ with $c=\left\langle\rho_{j}, e_{i}\right\rangle$, and also implies $\left\langle\rho_{i}, e^{\prime}\right\rangle=0$. Thus, (17) holds, and so, one has the implication (iii) $\Rightarrow$ (ii).

The equivalence (iv) $\Leftrightarrow$ (i) holds by Corollary 3.3 .

Definition 4.5. To any Lie algebra $L$ as before we associate a directed graph $\Gamma_{r}=\Gamma_{r}(L)$ on $r$ vertices $\left\{L_{i}\right\}_{i=1, \ldots, r}$, where a directed edge $\left[L_{j} \rightarrow L_{i}\right]$ joins the vertices $L_{i}$ and $L_{j}$ if and only if $\left\langle\rho_{j}, e_{i}\right\rangle>0$ for some $e_{i} \in R_{i}$.

Thus, there is no edge joining the vertices $L_{i}$ and $L_{j}$ of $\Gamma_{r}$ if and only if $\left[L_{i}, L_{j}\right]=0$, that is, the Lie algebra Lie $\left(L_{i}, L_{j}\right)$ is abelian. Furthermore, $\Gamma_{r}$ has no bidirected edge if and only if $L$ has no 2-pseudo-cycle of root derivations. For instance, this holds for the following graph $\Gamma_{3}=\Gamma_{3}(L)$ associated with the Lie algebra $L$ from Example 4.2 .

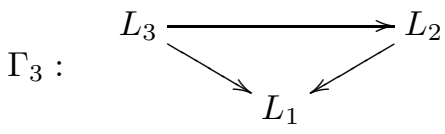

Lemma 4.6. The following are equivalent:

(i) $L$ contains no pseudo-cycle of root derivations;

(ii) $L$ contains no cycle of root derivations;

(iii) $L$ contains no 2-cycle of root derivations.

Proof. It suffices to prove (iii) $\Rightarrow(\mathrm{i})$, the two other implications being immediate.

Assume $L$ contains no 2-cycle, and then also no 2-pseudo-cycle of root derivations, see Lemma 4.4, Suppose to the contrary that $L$ has a pseudo-cycle of root derivations $\mathcal{D}=\left\{D_{1}, \ldots, D_{N}, D_{N+1}\right\}$ with $N \geq 3$. Then $\Gamma_{r}$ has the oriented cycle

$$
L_{\rho_{j(1)}} \rightarrow L_{\rho_{j(N)}} \rightarrow \ldots \rightarrow L_{\rho_{j(2)}} \rightarrow L_{\rho_{j(1)}}
$$

The sequence $\rho_{j(1)}, \ldots, \rho_{j(N)}$ of the corresponding ray generators can eventually contain repetitions. However, it is possible to subtract a subsequence $\rho_{j(1)}, \ldots, \rho_{j(t)}$ without repetition, where $3 \leq t \leq N$, such that $\rho_{j(t+1)}=$ $\rho_{j(1)}$. Then $\mathcal{D}^{\prime}=\left\{D_{1}, \ldots, D_{t}, D_{t+1}\right\}$ is again a pseudo-cycle, and the cycle

$$
L_{\rho_{j(1)}} \rightarrow L_{\rho_{j(t)}} \rightarrow \ldots \rightarrow L_{\rho_{j(2)}} \rightarrow L_{\rho_{j(1)}}
$$


has no self-intersection. To any $e \in R$ we associate the integer vector of length $t$,

$$
v(e)=\left(\left\langle\rho_{j(1)}, e\right\rangle, \ldots,\left\langle\rho_{j(t)}, e\right\rangle\right) \in \mathbb{Z}^{t} .
$$

One has

$$
\begin{aligned}
v\left(e_{j(1), 1}\right) & =(-1, \bullet, *, \ldots, *, *) \\
v\left(e_{j(2), 2}\right) & =(0,-1, \bullet, *, \ldots, *) \\
v\left(e_{j(3), 3}\right) & =(*, 0,-1, \bullet, \ldots, *) \\
& \vdots \\
v\left(e_{j(t-1), t-1}\right) & =(*, *, \ldots, 0,-1, \bullet) \\
v\left(e_{j(t), t}\right) & =(\bullet, *, *, \ldots, 0,-1)
\end{aligned}
$$

The stars in (22) stand for nonnegative integers, the bullets stand for positive integers, and the zeros on the lower subdiagonal are due to (16) and (17). In fact, (16) and (17) imply

$$
\left\langle\rho_{j(i)}, e\right\rangle=0 \quad \forall e \in R_{j(i+1)} .
$$

From (11), (16) and (23) one deduces

$$
\left\langle\rho_{j(i+2)}, e_{j(i), i}^{\prime}\right\rangle>0 \quad \text { where } \quad e_{j(i), i}^{\prime}:=e_{j(i), i}+e_{j(i+1), i+1} \in R_{j(i)}, i=1, \ldots, t-2 .
$$

Then (17) gives

$$
\left\langle\rho_{j(i)}, e\right\rangle=0 \quad \forall e \in R_{j(i+2)} .
$$

This means that the second lower subdiagonal in (22) consists of zeros. In the same fashion one can show that the third lower subdiagonal in (22) consists of zeros. Finally, we arrive by recursion to the conclusion that the matrix in (22) is upper triangular. Moreover, one has

$$
\left\langle\rho_{j(t+1)}, e\right\rangle=\left\langle\rho_{j(1)}, e\right\rangle=0 \quad \forall e \in R_{j(t)} .
$$

The latter contradicts (16) for $i=t$ and $e=e_{j(t), t}$.

The following statement strengthens Theorem 5.1 in [Arzhantsev et al., 2021] in application to our (simpler) setup. For the convenience of the reader we provide a complete proof, which exploits Lemma 4.6

Proposition 4.7. Assume $L$ contains no 2-cycle of root derivations. Then the associated graph $\Gamma_{r}$ is acyclic, that is, does not contain any oriented cycle, and the Lie algebra $L$ is finite-dimensional and nilpotent.

Proof. We freely use the notation from the proof of Lemma 4.6. Consider the one-dimensional Lie subalgebras $l_{\rho_{i}, e_{i}}$ of $L_{i}$ generated by the root derivations, where

$$
l_{\rho_{i}, e_{i}}=\operatorname{span}\left(\partial_{\rho_{i}, e_{i}}\right)=\mathbf{k} \partial_{\rho_{i}, e_{i}} \quad \text { with } \quad e_{i} \in R_{i} .
$$

Since $L$ has no 2-cycle then (17) holds. Hence, for $i \neq j$ there is the alternative:

$$
\text { either }\left[l_{\rho_{i}, e_{i}}, l_{\rho_{j}, e_{j}}\right]=0, \quad \text { or }\left[l_{\rho_{i}, e_{i}}, l_{\rho_{j}, e_{j}}\right] \in\left\{l_{\rho_{i}, e_{i}+e_{j}}, l_{\rho_{j}, e_{i}+e_{j}}\right\} \text {. }
$$

Due to (1) one has

$$
\left[l_{\rho_{i}, e_{i}}, l_{\rho_{j}, e_{j}}\right]=l_{\rho_{i}, e_{i}+e_{j}} \quad \text { if and only if }\left\langle\rho_{j}, e_{i}\right\rangle>0 .
$$

In the latter case $\Gamma_{r}$ contains the directed edge $\left[L_{j} \rightarrow L_{i}\right]$. It is clear that

$$
L_{i}=\bigoplus_{e \in R_{i}} l_{\rho_{i}, e} \text { and } L=\bigoplus_{i=1}^{r} L_{i}
$$

Therefore, one has

$$
\operatorname{dim}(L)=\sum_{i=1}^{r} \operatorname{dim}\left(L_{i}\right)=\sum_{i=1}^{r} \operatorname{card}\left(R_{i}\right)=\operatorname{card}(R) .
$$

Let us show that under our assumptions $\Gamma_{r}$ is acyclic, that is, does not contain any oriented cycle. Indeed, given an oriented cycle in $\Gamma_{r}$,

$$
L_{j(1)} \rightarrow L_{j(2)} \rightarrow \ldots \rightarrow L_{j(t)} \rightarrow L_{j(t+1)}=L_{j(1)}
$$


one can find a sequence of roots $e_{j(i), i} \in R_{j(i)}$ such that, with the usual convention $\rho_{j(t+1)}=\rho_{j(1)}$, one has

$$
\left\langle\rho_{j(i+1)}, e_{j(i), i}\right\rangle>0, \quad i=1, \ldots, t .
$$

Thus, $\mathcal{D}=\left(D_{i}=\partial_{\rho_{j(i)}, e_{j(i), i}}\right)_{i=1, \ldots, t}$ is a pseudo-cycle of root derivations in L. By Lemma 4.6, the latter contradicts our assumption on absence of 2-cycles in $L$.

A vertex $L_{i}$ is called a sink if either $L_{i}$ is isolated in $\Gamma_{r}$, or all the incident edges of $\Gamma_{r}$ at $L_{i}$ have the incoming direction, that is, $L_{i}$ does not emit any edge. The vertex $L_{i}$ of $\Gamma_{r}$ is a sink if and only if $L_{i}$ is an ideal of the Lie algebra $L$.

The end vertex of any maximal oriented path in $\Gamma_{r}$ is a sink. Since $\Gamma_{r}$ is acyclic it has at least one sink. Moreover, any connected component of $\Gamma_{r}$ contains a sink.

We can choose a new enumeration of the vertices of $\Gamma_{r}$ taking for $L_{1}$ a vertex which is a sink of $\Gamma_{r}$. Deleting $L_{1}$ from $\Gamma_{r}$ along with its incident edges yields a directed graph $\Gamma_{r-1}$. The corresponding Lie subalgebra of $L$ still has no pseudo-cycle of root derivations. Hence, $\Gamma_{r-1}$ has at least one sink. We choose a sink of $\Gamma_{r-1}$ to be $L_{2}$, etc. By construction, with this new enumeration one has (cf. Example 4.2)

$$
\begin{aligned}
{\left[L_{i}, L_{1}\right] } & \subset L_{1}, \quad i=2, \ldots, r, \\
{\left[L_{i}, L_{2}\right] } & \subset L_{2}, \quad i=3, \ldots, r, \\
\vdots & \\
{\left[L_{r}, L_{r-1}\right] } & \subset L_{r-1}, \\
{\left[L_{r}, L_{r}\right] } & =0 .
\end{aligned}
$$

To show that $L$ is of finite dimension, we use the enumeration of the subalgebras $L_{j} \subset L$ satisfying (27). We establish that the total coordinates of the vectors in $R$ are uniformly bounded above, and so, $R$ is finite. Due to (26) this yields the result.

At the beginning of Section 4 we defined $L$ as the Lie algebra generated by the finite set of root derivations $\partial_{i}, i=1, \ldots, s$. Given a ray generator $\rho_{j}$, consider all the root derivations $\partial_{i}$ among $\partial_{1}, \ldots, \partial_{s}$ which belong to $\rho_{j}$, and let $R_{j}^{(0)} \subset R_{j}$ be the set of their roots. It follows from (24) and (27) that $R_{r}=R_{r}^{(0)}$, and so, $R_{r}$ is finite. Furthermore, by (18), (20), and (27) for any $e \in R_{r}$ one has

$$
\left\langle\rho_{i}, e\right\rangle=0 \quad \forall i=1, \ldots, r-1 \quad \text { and } \quad\left\langle\rho_{r}, e\right\rangle=-1 .
$$

Again by (24) and (27), any root $e \in R_{r-1}$ is of the form

$$
e=e_{r-1}^{(0)}+e_{r, 1}+\ldots+e_{r, m} \quad \text { with } \quad e_{r-1}^{(0)} \in R_{r-1}^{(0)} \quad \text { and } \quad e_{r, i} \in R_{r}, i=1, \ldots, m,
$$

where the lattice vectors $e_{r, i} \in R_{r}$ are not necessarily distinct. We claim that

$$
0 \leq m \leq\left\langle\rho_{r}, e_{r-1}^{(0)}\right\rangle .
$$

Indeed, for the $r$ th total coordinate of the lattice vectors in (28) we have

$$
\left\langle\rho_{r}, e_{r, i}\right\rangle=-1, i=1, \ldots, m, \quad \text { and }\left\langle\rho_{r}, e\right\rangle=\left\langle\rho_{r}, e_{r-1}^{(0)}\right\rangle-m \geq 0 .
$$

Since both $R_{r-1}^{(0)}$ and $R_{r}$ are finite, we conclude that $R_{r-1}$ is as well.

Suppose by induction that the $R_{i}$ are finite for $i=t, \ldots, r$, where $2 \leq t \leq r-1$. By (24) and (27), any root $e \in R_{t-1}$ is of the form

$$
e=e_{t-1}^{(0)}+\sum_{i=t}^{r} \sum_{j=1}^{m_{i}} e_{i, j} \quad \text { with } \quad e_{t-1}^{(0)} \in R_{t-1}^{(0)} \quad \text { and } \quad e_{i, j} \in R_{i},
$$

with possible repetitions of the summands. Likewise in (22), due to the chosen enumeration we obtain for the first $i$ total coordinates of the vector $e_{i, j} \in R_{i}$,

$$
\left\langle\rho_{l}, e_{i, j}\right\rangle=0 \quad \forall l=1, \ldots, i-1 \quad \text { and } \quad\left\langle\rho_{i}, e_{i, j}\right\rangle=-1, j=1, \ldots, m_{i} .
$$

Letting $l=t$ yields

$$
\left\langle\rho_{t}, e_{i, j}\right\rangle=\left\{\begin{aligned}
0, & i=t+1, \ldots, r \\
-1, & i=t
\end{aligned}\right.
$$


From (29)-31) we deduce

$$
\left\langle\rho_{l}, e\right\rangle=0 \quad \forall l<t-1, \quad\left\langle\rho_{t-1}, e\right\rangle=-1, \quad \text { and } \quad\left\langle\rho_{t}, e\right\rangle=\left\langle\rho_{t}, e_{t-1}^{(0)}\right\rangle-m_{t} \geq 0 .
$$

Therefore, one has

$$
m_{t} \leq\left\langle\rho_{t}, e_{t-1}^{(0)}\right\rangle=: \tilde{m}_{t}
$$

To find a uniform bound for the $(t+1)$ st total coordinate $\left\langle\rho_{t+1}, e\right\rangle$ of $e$ we let

$$
\tilde{m}_{t+1}=\max _{e_{t-1}^{(0)} \in R_{t-1}^{(0)}}\left\{\left\langle\rho_{t+1}, e_{t-1}^{(0)}\right\rangle\right\}+\max _{m_{t} \leq \tilde{m}_{t}}\left\{\sum_{j=1}^{m_{t}}\left\langle\rho_{t+1}, e_{t, j}\right\rangle \mid\left(e_{t, 1}, \ldots, e_{t, m_{t}}\right) \in R_{t}^{m_{t}}\right\}<+\infty .
$$

Arguing as before we obtain $m_{t+1} \leq \tilde{m}_{t+1}$. Continuing in this way we arrive at the conclusion that all the total coordinates of the vectors $e$ from $R_{t-1}$ are uniformly bounded above, and so, $R_{t-1}$ is finite. This gives the induction step. Thus, $R$ is finite, and the dimension $\operatorname{dim}(L)=\operatorname{card}(R)$ is finite too, see (26).

Let us show finally that $L$ is nilpotent. Indeed, let $1 \leq i<j \leq r$. Using the relations similar to (30) and the fact that $R$ is finite, for $N \gg 1$ one deduces

$$
\left\langle\rho_{j}, e+e_{1}+\ldots+e_{N}\right\rangle=\left\langle\rho_{j}, e\right\rangle-N \leq-2 \quad \text { whenever } \quad e \in R_{i} \quad \text { and } \quad e_{1}, \ldots, e_{N} \in R_{j} .
$$

Letting $l=\mathbf{k} \partial_{\rho_{i}, e} \subset L_{i}$ and $l_{k}=\mathbf{k} \partial_{\rho_{j}, e_{k}} \subset L_{j}, k=1, \ldots, N$ and using (25) we obtain by (32)

$$
\left[l_{1},\left[l_{2},\left[\ldots,\left[l_{N}, l\right] \ldots\right]=0 \quad \text { whenever } \quad l_{k} \subset L_{j}, k=1, \ldots, N, \quad \text { and } \quad l \subset L_{i}, \quad i \leq j .\right.\right.
$$

For $N \gg 1$ the latter vanishing reads

$$
\operatorname{ad}\left(L_{j}\right)^{N}\left(L_{i}\right)=0 \quad \forall j \geq i, \quad i, j \in\{1, \ldots, r\} .
$$

Taking into account (27), from (33) we deduce

$$
\operatorname{ad}(L)^{N r}(L)=0
$$

which means that $L$ is nilpotent.

\subsection{From nilpotent Lie algebras to unipotent groups}

It is well known, see, e.g., Hochschild, 1981, Ch. XVI, Thm. 4.2], that over a field of characteristic zero, the Lie functor realizes the equivalence between the categories of unipotent algebraic groups and of nilpotent Lie algebras. In our particular case, this correspondence can be made quite explicit.

Proposition 4.8. Let $X$ be a toric affine variety over $\mathbf{k}$ with no torus factor, let $G=\left\langle U_{1}, \ldots, U_{s}\right\rangle \subset$ Aut $(X)$ be a subgroup generated by the root subgroups $U_{i}=\exp \left(t \partial_{i}\right)$, where the $\partial_{i}$ are locally nilpotent derivations of the structure algebra $\mathcal{O}(X)$ associated with Demazure roots, let $L$ be the Lie algebra generated by $\partial_{1}, \ldots, \partial_{s}$, and let $\Gamma(L)=\Gamma_{r}(L)$ be the associated directed graph, see Definition 4.5. Then the following are equivalent:

(i) $L$ has no 2-cycle of root derivations;

(ii) the graph $\Gamma(L)$ has no oriented cycle, in particular, no bidirected edge;

(iii) $L$ is finite-dimensional and nilpotent;

(iv) $G$ is a unipotent algebraic group acting regularly on $X$.

In the latter case one has $L=\operatorname{Lie}(G)$.

Proof. The implications (i) $\Rightarrow$ (ii)\&(iii) follow from Proposition 4.7 Condition (iii) implies (iv) of Lemma 4.4 and so, by virtue of this lemma, implies (i). Therefore, there is the equivalence (i) $\Leftrightarrow$ (iii). By Definition 4.5 . $\Gamma(L)$ has no bidirected edge if and only if $L$ has no 2-pseudo-cycle of root derivations. By virtue of Lemma 4.4 this is equivalent to (i). Thus, one has (i) $\Leftrightarrow$ (ii) $\Leftrightarrow$ (iii).

If (iv) holds, then $G$ is a nilpotent group, and so, it contains no non-abelian free subgroup. This implies (i) due to Proposition 3.1 and Corollary 3.3 . Hence, we have the implications (iv) $\Rightarrow$ (i) $\Leftrightarrow$ (iii). The converse implication (iii) $\Rightarrow$ (iv) is proven in Lemmas 4.94 .12 below. 
Convention. We assume in the sequel that the Lie algebra $L$ is finite-dimensional and nilpotent, and so, (i)-(iii) hold. We use the enumeration of the subalgebras $L_{i} \subset L, i=1, \ldots, r$ introduced in the proof of Proposition 4.7 . so that (27) and (33) hold.

Let us recollect the notation. Recall that $k$ stands for the number of total coordinates on our toric affine variety $X$; this is at the same time the number of extremal rays $\rho_{i}$ of the cone $\Delta_{\mathbb{Q}}$, see subsections 2.1] and 2.3. Due to (5), any automorphism of $X$ lifts to an automorphism of the Cox $\operatorname{ring} \mathcal{O}\left(\mathbb{A}^{k}\right)=\mathbf{k}\left[x_{1}, \ldots, x_{k}\right]$ of $X$, and to an automorphism of the spectrum $\mathbb{A}^{k}$ of the Cox ring. The algebra $\mathcal{O}(X)$ coincides with the algebra of the $F_{\mathrm{Cox}}$-invariants of the Cox ring $\mathcal{O}\left(\mathbb{A}^{k}\right)$. By Lemma 2.1. b any root derivation $\partial_{i}, i=1, \ldots, s$, lifts to a root derivation $\hat{\partial}_{i}$ of $\mathbf{k}\left[x_{1}, \ldots, x_{k}\right]$ of form (2), and any root subgroup $U_{i}=\exp \left(t \partial_{i}\right)$ lifts to a root subgroup $\hat{U}_{i}=\exp \left(t \hat{\partial}_{i}\right)$ consisting of elementary transformations (3i) and centralized by the Cox quasitorus $F_{\text {Cox }}$. The derivation $\partial_{i}$ is the restriction of $\hat{\partial}_{i}$ to $\mathcal{O}\left(\mathbb{A}^{k}\right)^{F_{\mathrm{Cox}}} \cong \mathcal{O}(X), i=1, \ldots, s$. This yields an isomorphism $L \cong \hat{L}$, where $\hat{L}$ is the Lie algebra generated by $\hat{\partial}_{1}, \ldots, \hat{\partial}_{s}$, see Lemma 2.1]d.

Assuming that $\hat{G}=\left\langle\hat{U}_{1}, \ldots, \hat{U}_{s}\right\rangle$ is a unipotent linear algebraic group, one has an isomorphism $G \cong \hat{G}$, and so, $G$ is a unipotent linear algebraic group too, see Proposition 2.2 b. Thus, it suffices to show that $\hat{G}$ is a unipotent linear algebraic group provided $\hat{L}$ is a finite-dimensional nilpotent Lie algebra.

Let $\hat{L}_{i}$ be the image of $L_{i}$ under the isomorphism $L \cong \hat{L}$ from Lemma 2.1. d. It is easily seen that $L$ satisfies (i)-(iii) if and only if $\hat{L}$ does.

Recall that the automorphisms of $\mathbb{A}^{k}$ of the form

$$
\left(x_{1}, \ldots, x_{k}\right) \mapsto\left(x_{1}+f_{1}, \ldots, x_{k}+f_{k}\right), \text { where } f_{i} \in \mathbf{k}\left[x_{i+1}, \ldots, x_{k}\right], \quad i=1, \ldots, k,
$$

are called unitriangular. These automorphisms form the unitriangular subgroup of the group $\operatorname{Aut}\left(\mathbb{A}^{k}\right)$, see Freudenburg, 2017, Ch. 3]. In Lemmas 4.9 and 4.10 we present $\hat{G}=\left\langle\hat{U}_{1}, \ldots, \hat{U}_{s}\right\rangle$ as a subgroup of the unitriangular group with $\operatorname{Lie}(\hat{G})=\hat{L}$.

Lemma 4.9. Any $\partial \in L$ is a locally nilpotent derivation of $\mathcal{O}(X)$.

Proof. Due to (21), in the total coordinates $\left(x_{1}, \ldots, x_{k}\right)$ any derivation $\hat{\partial} \in \hat{L}_{i}, i \in\{1, \ldots, r\}$, acts on $\mathbb{A}^{k}$ via

$$
\hat{\partial}=p \partial / \partial x_{i} \quad \text { where } \quad p \in \mathbf{k}\left[x_{1}, \ldots, x_{i-1}, x_{i+1}, \ldots, x_{k}\right] .
$$

The monomials $M_{j}$ in (2), taken up to proportionality, of all possible polynomials $p$ in (35) are in one-to-one correspondence with the roots in the subset $R_{j}$ of $R$. Since $R_{j}$ is finite, it follows that

$$
\max _{\hat{\partial} \in \hat{L}_{j}}\{\operatorname{deg}(p)\} \leq d_{j} \quad \text { for some } \quad d_{j} \in \mathbb{N} .
$$

Due to our choice of enumeration, the subalgebras $\hat{L}_{i} \subset \hat{L}$ satisfy (27). Hence, the total coordinates of the roots in $R_{i}$ form a triangular-like matrix, cf. (30), that is, $p \in \mathbf{k}\left[x_{i+1}, \ldots, x_{k}\right]$ for any $p$ in (35). Since $\hat{\partial}^{2}\left(x_{i}\right)=0$, $i=1, \ldots, k$, the $\mathbb{G}_{a}$-subgroup $\exp (t \hat{\partial})$ of $\operatorname{Aut}\left(\mathbb{A}^{k}\right)$ generated by $\hat{\partial}$ from (35) acts on $\mathbb{A}^{k}$ via the unitriangular (elementary) transformations

$$
\exp (t \hat{\partial}):\left(x_{1}, \ldots, x_{k}\right) \mapsto\left(x_{1}, \ldots, x_{i-1}, x_{i}+t p\left(x_{i+1}, \ldots, x_{k}\right), x_{i+1}, \ldots, x_{k}\right), \quad t \in \mathbf{k},
$$

cf. (3). More generally, any derivation $\hat{\partial} \in \hat{L}$ is triangular of the form

$$
\hat{\partial}=\sum_{i=1}^{r} \delta_{i}=\sum_{i=1}^{r} p_{i} \partial / \partial x_{i}, \quad \text { where } \quad \delta_{i} \in \hat{L}_{i} \quad \text { and } \quad p_{i} \in \mathbf{k}\left[x_{i+1}, \ldots, x_{k}\right], i=1, \ldots, r .
$$

According to Freudenburg, 2017, Prop. 3.29], $\hat{\partial}$ is locally nilpotent on $\mathcal{O}\left(\mathbb{A}^{k}\right)$, and then also $\partial$ is locally nilpotent on $\mathcal{O}(X)=\mathcal{O}\left(\mathbb{A}^{k}\right)^{F_{\text {Cox }}}$.

Notice that for $r<k$ the variables $x_{r+1}, \ldots, x_{k}$ belong to the kernel of any derivation $\hat{\partial} \in \hat{L}$.

Classical formulas. In what follows we use the classical Baker-Campbell-Hausdorff (BCH) and Zassenhaus formulas. Let us recall these formulas following Bonfiglioli et al., 2012, Li et al., 2019, Manetti, 2012, Wang et al., 2019]. Let $A$ be an associative algebra with unit over k. In the formal power series algebra $A[[t]]$ the function exp is well defined for any series without constant term, and log is well defined for any series with the constant term equal 1. For $a, b \in A$ consider the Lie subalgebra $\operatorname{Lie}(a, b)$ of $A$, that is, the 
smallest subspace of $A$ which contains $a$ and $b$ and is stable under commutators. The BCH formula expresses $c(t):=\log (\exp (t a) \exp (t b)) \in \operatorname{Lie}(a, b)[[t]]$. Plugging in formally $t=1$, the formula reads:

$$
c:=c(1)=a+b+\frac{1}{2}[a, b]+\frac{1}{12}[a,[a, b]]+\frac{1}{12}[b,[b, a]]+\ldots,
$$

where each term is a rational multiple of the Lie monomial in $a$ and $b$ of degree $m$ obtained from the universal Lie monomial $\alpha\left[z_{1},\left[z_{2},\left[\ldots, z_{m}\right] \ldots\right], \alpha \in \mathbb{Q}\right.$, after substitution of either $a$ or $b$ instead of every $z_{i}$.

Likewise, for given $a_{1}, \ldots, a_{\nu} \in A$, the multivariate Zassenhaus formula reads:

$$
\exp \left(a_{1}+\ldots+a_{\nu}\right)=\exp \left(a_{1}\right) \cdots \exp \left(a_{\nu}\right) \prod_{m=2}^{\infty} \exp \left(\psi_{m}\left(a_{1}, \ldots, a_{\nu}\right)\right)
$$

where $\psi_{m}\left(a_{1}, \ldots, a_{\nu}\right)$ is a homogeneous Lie polynomial in $a_{1}, \ldots, a_{\nu}$ of degree $m$. Notice that this description applies to any Lie algebra $\mathfrak{g}$ and its universal enveloping algebra $A$.

In our setup, it occurs that the both formulas contain just a finite number of terms, and, respectively, factors. Indeed, letting $L=\operatorname{Lie}(a, b)$ in the former case and $L=\operatorname{Lie}\left(a_{1}, \ldots, a_{\nu}\right)$ in the latter case, suppose that $L$ is nilpotent of nilpotency class $n$, that is, $L^{n+1}=\operatorname{ad}(L)^{n}(L)=0$, where $n \in \mathbb{N}$ is minimal with this property. Then the homogeneous Lie polynomials of degree $m>n$ in the both formulas vanish, hence the sum in (39) and the product in (40) are finite.

Lemma 4.10. In the total coordinates, the automorphisms $\exp (\hat{L})=\{\exp (\hat{\partial}) \mid \hat{\partial} \in \hat{L}\}$ form a group of unitriangular automorphisms of $\mathbb{A}^{k}$. The map $\exp : \hat{L} \rightarrow \exp (\hat{L})$ is well defined and bijective.

Proof. By virtue of (37) and (38), any automorphism $\exp (\hat{\partial}) \in \exp (\hat{L}) \subset \operatorname{Aut}\left(\mathbb{A}^{k}\right)$ is unitriangular of the form

$$
\exp (\hat{\partial}):\left(x_{1}, \ldots, x_{k}\right) \mapsto\left(x_{1}+f_{1}, \ldots, x_{r}+f_{r}, x_{r+1}, \ldots, x_{k}\right) \text { with } f_{i} \in \mathbf{k}\left[x_{i+1}, \ldots, x_{k}\right]
$$

Any unitriangular automorphism $\alpha \in \operatorname{Aut}\left(\mathbb{A}^{k}\right)$ can be written as the exponential $\alpha=\exp (c)$ of the triangular derivation

$$
c=\log (\alpha)=\log (\mathrm{id}+(\alpha-\mathrm{id})) \in \operatorname{Der}\left(\mathcal{O}\left(\mathbb{A}^{k}\right)\right),
$$

see [Freudenburg, 2017, Prop. 3.30] and its proof. Consider a pair $(a, b)$ of triangular derivations of $\mathcal{O}\left(\mathbb{A}^{k}\right)$ from $\hat{L}$. The product $\exp (a) \exp (b)$ of the corresponding unitriangular automorphisms is again unitriangular. In more detail, $\exp (a) \exp (b)=\exp (c)$ with a triangular derivation

$$
c=\log (\exp (a) \exp (b)) \in \operatorname{Der}\left(\mathcal{O}\left(\mathbb{A}^{k}\right)\right)
$$

see Freudenburg, 2017, Cor. 3.31]. The latter derivation can be expressed via the BCH formula (39) truncated on level $n+1$, where $n$ is the nilpotency class of $\hat{L}$. It follows that $c \in \hat{L}$. Thus, $\exp (\hat{L})$ is a subgroup of the group of unitriangular automorphisms of $\mathbb{A}^{k}$. Since $\log$ and $\exp$ are mutually inverse, the $\operatorname{map} \exp : \hat{L} \rightarrow \exp (\hat{L})$ is a bijection.

Lemma 4.11. The degrees of $\exp (\hat{\partial})$ are uniformly bounded for $\hat{\partial} \in \hat{L}$.

Proof. Write $\hat{\partial}=a_{1}+\ldots+a_{\nu} \in \hat{L}$, where $a_{i} \in \hat{L}_{i}$. We can $\operatorname{express} \exp (\hat{\partial})=\exp \left(a_{1}+\ldots+a_{\nu}\right)$ via the Zassenhaus formula (40), where the product is truncated on level $n+1$ with $n$ being the nilpotency class of $\hat{L}$.

Consider the increasing chain of ideals of $\hat{L}$,

$$
\mathcal{L}_{1} \subset \mathcal{L}_{2} \subset \ldots \subset \mathcal{L}_{r}=\hat{L}, \quad \text { where } \quad \mathcal{L}_{\nu}:=\hat{L}_{1} \oplus \ldots \oplus \hat{L}_{\nu},
$$

see (27). Notice that $\hat{\partial}=a_{1}+\ldots+a_{\nu} \in \mathcal{L}_{\nu}$. Let us show that in (40) one has $\psi_{m}\left(a_{1}, \ldots, a_{\nu}\right) \in \mathcal{L}_{\nu-1}$ for any $\nu \in\{2, \ldots, r\}, m \geq 2$. Indeed, it suffices to check this for $\psi_{m}$ which is a Lie monomial in $a_{1}, \ldots, a_{\nu}$ of degree $m$. In this case, our claim follows from the fact that the abelian Lie algebras $\hat{L}_{1}, \ldots, \hat{L}_{r}$ verify (27).

Now we proceed by induction on $\nu=1, \ldots, r$. The assertion of the lemma holds for $\mathcal{L}_{1}$ due to (36) and (37). Suppose it holds for some $\mathcal{L}_{\nu-1}$. Take

$$
\hat{\partial}=\sum_{i=1}^{\nu} a_{i}=\sum_{i=1}^{\nu} p_{i} \partial / \partial x_{i} \in \mathcal{L}_{\nu}, \quad \text { where } \quad a_{i} \in \hat{L}_{i} .
$$


Using (40) and the preceding observation, we can write

$$
\exp (\hat{\partial})=g_{1} \exp \left(a_{\nu}\right) g_{2}=g_{1} \exp \left(p_{\nu} \partial / \partial x_{\nu}\right) g_{2}, \quad \text { where } \quad g_{1}, g_{2} \in \exp \left(\mathcal{L}_{\nu-1}\right)
$$

Since our assertion holds for $\exp \left(\mathcal{L}_{\nu-1}\right)$ by the induction hypothesis and for $\exp \left(\hat{L}_{\nu}\right)$ by (36) -(37), the degrees of all the automorphisms in (42) are uniformly bounded above. Therefore, the assertion holds for $\exp \left(\mathcal{L}_{\nu}\right)$ as well. This concludes the induction.

Lemma 4.12. Assume $L$ is a finite dimensional nilpotent Lie algebra. Then $G$ is a unipotent algebraic group acting regularly on $X$, and $L=\operatorname{Lie}(\mathrm{G})$.

Proof. Due to Lemma 4.11, the $\operatorname{span} F$ of $\exp (\hat{L})$ is a finite-dimensional subspace of the vector $\operatorname{space} \operatorname{End}\left(\mathbb{A}^{k}\right)$. One can take for the coordinates in $\hat{L}$ and $F$ the coefficients of the polynomials $p_{1}, \ldots, p_{r}$ and $f_{1}, \ldots, f_{r}$ in (38) and (41), respectively. The map

$$
\hat{L} \ni \hat{\partial} \mapsto \exp (\hat{\partial}) \in \exp (\hat{L}), \quad\left(p_{1}, \ldots, p_{r}\right) \mapsto\left(f_{1}, \ldots, f_{r}\right),
$$

defines a morphism of algebraic varieties $\hat{L} \rightarrow F$. The image $\exp (\hat{L})$ is an irreducible constructible subset of $F$. Since $\exp (\hat{L})$ is a connected group, this is a locally closed smooth subvariety of $F$. By Zariski's Main Theorem, the bijective morphism $\exp : \hat{L} \rightarrow \exp (\hat{L})$ of smooth varieties is an isomorphism. Since $\hat{L}$ is an affine variety, $\exp (\hat{L})$ is too. Using (34), it is easily seen that $\exp (\hat{L})$ is an affine algebraic group which acts regularly on $\mathbb{A}^{k}$. The exponential of a nilpotent matrix is unipotent. Therefore, the group $\exp (\hat{L})$ is unipotent since it consists of unipotent elements.

Recall that $\hat{G}=\left\langle\hat{U}_{1}, \ldots, \hat{U}_{s}\right\rangle$, where $\hat{U}_{j}=\exp \left(t \hat{\partial}_{j}\right)$ with $\hat{\partial}_{j} \in \hat{L}$. So, $\hat{U}_{j} \subset \exp (\hat{L})$ for any $j=1, \ldots, s$. It follows that $\hat{G} \subset \exp (\hat{L})$. In fact, $\hat{G}=\exp (\hat{L})$ because the Lie subalgebras $\operatorname{Lie}\left(\hat{U}_{j}\right)=\mathbf{k} \hat{\partial}_{j} \subset \hat{L}, j=1, \ldots, s$, generate $\hat{L}$. By the preceding discussion we deduce $\operatorname{Lie}(\hat{G})=\hat{L}$. It follows that $\operatorname{Lie}(G)=L$.

Proof of Proposition 4.1. Due to Corollary 3.3, under the assumption of Proposition 4.1. (17) holds for any $e \in R_{i}, e^{\prime} \in R_{j}$. Then $L$ has no 2-cycle of root derivations. Now the assertion follows from Propositions 4.7 and 4.8 .

\section{Transitive actions}

\subsection{Doubly transitive groups acting on toric affine varieties}

In this section we apply the Tits' type alternative to answer Question 1 under the assumption of double transitivity of the group in question. We start with the following simple combinatorial lemma.

Lemma 5.1. Let a group $G$ act effectively and doubly transitively on a set $X$, where $\operatorname{card}(X) \geq 3$. Then the following hold.

(a) Any nontrivial normal subgroup $H$ of $G$ is transitive on $X$;

(b) the stabilizer $G_{x}$ of a point $x \in X$ acts transitively on $X \backslash\{x\}$, and so, $x$ is a unique fixed point of $G_{x}$;

(c) the center of $G$ is trivial.

Proof. To show (a) it suffices to notice that $G$ permutes the $H$-orbits on $X$. Statement (b) is immediate. To show (c), assume that the center $Z$ of $G$ is nontrivial. Then by (a), $Z$ is transitive on $X$. On the other hand, since $Z$ commutes with $G_{x}$, it fixes the unique fixed point $x$ of $G_{x}$, see (b). This gives a contradiction.

The next proposition follows immediately from Lemma [5.1(c).

Proposition 5.2. No nilpotent group acts doubly transitively on a set $X$ with $\operatorname{card}(X) \geq 3$. In particular, a unipotent linear algebraic group cannot act 2-transitively on an algebraic variety.

Remark 5.3. Alternatively, the second statement can be deduced from the classification of doubly transitive groups of homeomorphisms and doubly transitive Lie groups, see [Kramer, 2003, Tits, 1952, 1956], or by using Humphreys, 1975, Prop. 17.4 and Cor. 17.5].

Now we can deduce Corollary 1.2 from the Introduction.

Proof of Corollary 1.2. The assertion follows immediately from Theorem 1.1 and Proposition 5.2 . 


\subsection{High transitivity of a subnormal subgroup}

As we indicated in the Introduction, the highly transitive actions of the groups generated by one-parameter unipotent subgroups were a starting point of this research. We add below some results concerning a combinatorial aspect of high transitivity, which could be useful in order to attack Conjecture 1 .

Definition 5.4. Let $G$ be a group. We say that $G$ is highly transitive if $G$ admits an effective action on a set $X$ which is $m$-transitive for any $m \in \mathbb{N}$.

Attention: one can find in the literature another definition of high transitivity, which does not require effectiveness.

The non-abelian free groups provide examples of highly transitive groups Cameron, 1987, McDonough et al., 1977]. Recall that a subgroup $N$ of a group $G$ is called subnormal if there exists a descending normal series

$$
G \unrhd N_{1} \unrhd N_{2} \unrhd \ldots \unrhd N_{k}=N .
$$

Proposition 5.5. Assume that a group $G$ acts effectively and highly transitively on an infinite set $X$. Then any nontrivial subnormal subgroup $N$ of $G$ is also highly transitive on $X$. In particular, $N$ cannot be virtually solvable.

The proof is done at the end of the section; it is based on the following lemma. In turn, the proof of the lemma imitates the one of Dixon et al., 1996, Cor. 7.2A]. For the sake of completeness, we provide the argument.

Lemma 5.6. Assume that a group $G$ acts effectively and highly transitively on a set $X$. Let $H$ be a nontrivial normal subgroup of $G$. Then $H$ acts on $X$ highly transitively.

Proof. For any $m$-tuple $\alpha=\left\{x_{1}, \ldots, x_{m}\right\}$ of pairwise distinct points in $X$ we consider the stabilizers

$$
G_{\alpha}=G_{x_{1}} \cap \ldots \cap G_{x_{m}} \quad \text { and } \quad H_{\alpha}=H_{x_{1}} \cap \ldots \cap H_{x_{m}} .
$$

Then $H_{\alpha}$ is a normal subgroup in $G_{\alpha}$. It suffices to show that for any positive integer $m$ and for any $m$-tuple $\alpha$ the group $H_{\alpha}$ acts transitively on $X \backslash\left\{x_{1}, \ldots, x_{m}\right\}$. Assuming the contrary, take the minimal $m \geq 1$ such that $H_{\alpha}$ is not transitive on $X \backslash\left\{x_{1}, \ldots, x_{m}\right\}$. Notice that, according to Lemma 5.1(a), for $m=0$ the group $H_{\alpha}=H$ is transitive on $X \backslash\left\{x_{1}, \ldots, x_{m}\right\}=X$.

By assumption, $G_{\alpha}$ acts highly transitively on $X \backslash\left\{x_{1}, \ldots, x_{m}\right\}$. From Lemma [5.1(a) we deduce that $H_{\alpha}=\{e\}$.

Let $\beta=\left\{x_{1}, \ldots, x_{m-1}\right\}$. By the minimality of $m$, the stabilizer $H_{\beta}$ is transitive on $X \backslash\left\{x_{1}, \ldots, x_{m-1}\right\}$. From $H_{\alpha}=\{e\}$ it follows that $H_{\beta}$ is simply transitive on $X \backslash\left\{x_{1}, \ldots, x_{m-1}\right\}$. So, we can identify the set $X \backslash\left\{x_{1}, \ldots, x_{m}\right\}$ with $H_{\beta} \backslash\{e\}$ via the bijection

$$
X \backslash\left\{x_{1}, \ldots, x_{m}\right\} \ni y \mapsto h \in H_{\beta} \backslash\{e\}, \quad \text { where } y=h x_{m} .
$$

Under this identification, the (highly transitive) action of $G_{\alpha}$ on $X \backslash\left\{x_{1}, \ldots, x_{m}\right\}$ corresponds to the action of $G_{\alpha}$ by conjugation on $H_{\beta} \backslash\{e\}$. Indeed, the latter is due to the relation

$$
g h x_{m}=g h g^{-1} g x_{m}=g h g^{-1} x_{m} \quad \forall g \in G_{\alpha}, \quad \forall h \in H_{\beta} \backslash\{e\} .
$$

The action by conjugation sends a pair $\left(h, h^{-1}\right)$ to a pair of the same type. Since $H_{\beta}$ is infinite, it follows that the action of $G_{\alpha} \subset \operatorname{Aut}\left(H_{\beta}\right)$ on $H_{\beta} \backslash\{e\}$ cannot be 2-transitive, unless $H_{\beta}$ is a group of exponent two.

Suppose finally that $H_{\beta}$ is a group of exponent two. It is well known that $H_{\beta}$ is a power of $\mathbb{Z} / 2 \mathbb{Z}$, or, in other words, the additive group of a vector space $V$ over the field $\mathbb{F}_{2}$ with two elements. However, the action of $\operatorname{Aut}\left(H_{\beta}\right)=\mathrm{GL}(V)$ is not 3-transitive on $H_{\beta} \backslash\{e\}=V \backslash\{0\}$ contrary to our assumption, because it preserves the linear (in)dependence. This contradiction completes the proof.

Remark 5.7. Notice that the affine group $G=\operatorname{Aff}(V)$ of the vector space $V=\mathbb{A}_{\mathbb{F}_{2}}^{n}, n \geq 3$, acts 3-transitively on $V$, while the normal subgroup of translations acts just simply transitively on $V$, contrary to Dixon et al., 1996, Exercise 2.1.16].

Proof of Proposition 5.5. The first assertion of 5.5 follows from Lemma 5.6 by recursion on the length of the normal series (43). As for the second assertion, notice that any virtually solvable group $G$ contains a normal solvable subgroup $H$ of finite index. In turn, $H$ contains a nontrivial normal abelian subgroup $A$, and $A$ contains a nontrivial cyclic subgroup, say, $N$, which is a subnormal subgroup of $G$. However, a cyclic group cannot be highly transitive. 
Remark 5.8. Due to Gromov's Theorem Gromov, 1981], a finitely generated group has polynomial growth if and only if it is virtually nilpotent. Hence, by Proposition 5.5 no nontrivial subnormal finitely generated subgroup of a highly transitive group has polynomial growth.

\section{Funding}

The work of the first author was supported by the grant RSF-19-11-00172.

\section{Acknowledgements}

The second author is grateful to François Dahmani, Rostislav Grigorchuk, Alexei Kanel-Belov, Boris Kunyavski, Stéphane Lamy, Bertrand Remy, and Christian Urech for useful conversations, letter exchange, and references. Our thanks are due as well to a referee for fruitful remarks and suggestions, which allowed to improve essentially the presentation and to strengthen one of the main results.

\section{References}

Andrist, R. B. "Integrable generators of Lie algebras of vector fields on $\mathbb{C}^{n}$." Forum Math. 31 (2019):943-949.

Arzhantsev, I., Derenthal, U., Hausen, J., and Laface, A. Cox rings. Cambridge Studies in Advanced Mathematics, 144. Cambridge University Press, Cambridge, 2015.

Arzhantsev, I., Flenner, H., Kaliman, S., Kutzschebauch, F., and Zaidenberg, M. "Flexible varieties and automorphism groups." Duke Math. J. 162 (2013):767-823.

Arzhantsev, I. and Gaifullin, S. "Cox rings, semigroups, and automorphisms of affine algebraic varieties." Sb. Math. 201 (2010):1-21.

Arzhantsev, I., Kuyumzhiyan, K., and Zaidenberg, M. "Flag varieties, toric varieties, and suspensions: three instances of infinite transitivity." Sb. Math. 203 (2012):3-30.

Arzhantsev, I., Kuyumzhiyan, K., and Zaidenberg, M. "Infinite transitivity, finite generation, and Demazure roots." Adv. Math. 351 (2019):1-32.

Arzhantsev, I., Liendo, A., and Stasyuk, T. "Lie algebras of vertical derivations on semiaffine varieties with torus actions." J. Pure Appl. Algebra 225 (2021):106499.

Bisi, C., Furter, J.-Ph., and Lamy, S. "The tame automorphism group of an affine quadric threefold acting on a square complex." J. Éc. polytech. Math. 1 (2014):161-223.

Bonfiglioli, A., and Fulci, R. Topics in noncommutative algebra. The theorem of Campbell, Baker, Hausdorff and Dynkin. Lecture Notes in Mathematics, 2034. Springer, Heidelberg, 2012.

Cameron, P. J. "Some permutation representations of a free group." Europ. J. Combin. 8 (1987):257-260.

Campana, F., Wang, Fei, and Zhang, De-Qi. "Automorphism groups of positive entropy on projective threefolds." Trans. Amer. Math. Soc. 366 (2014):1621-1638.

Cantat, S. "Sur les groupes de transformations birationnelles des surfaces." Ann. of Math. (2) 174 (2011):299-340.

Cantat, S. "Sur les groupes de transformations birationnelles des surfaces (version longue)." 52 pp. Available at: https://perso.univ-rennes1.fr/serge.cantat/Articles/cremona_long.pdf

Cantat, S. "The Cremona group." Algebraic geometry: Salt Lake City 2015, 101-142. Proc. Sympos. Pure Math., 97.1. Amer. Math. Soc., Providence, RI, 2018.

Chistopolskaya, A. "On nilpotent generators of the Lie algebra $\mathfrak{s l}_{n}$." Linear Algebra Appl. 559 (2018):73-79.

Cox, D. A., Little J. B., and Schenck, H. K. Toric Varieties. Graduate Studies in Mathematics, 124. Amer. Math. Soc., Providence, RI, 2011.

Demazure, M. Sous-groupes algébriques de rang maximum du groupe de Cremona. Ann. Sci. Éc. Norm. Supér. (4), 3:(1970), 507-588.

Dicks, W. "Automorphisms of the polynomial ring in two variables." Publ. Sec. Mat. 27 (1983):155-162. 
Dinh, Tien-Cuong. "Tits alternative for automorphism groups of compact Kähler manifolds." Acta Math. Vietnam. 37(4) (2012):513-529.

Dinh, Tien-Cuong, Hu, Fei, and Zhang, De-Qi. "Compact Kähler manifolds admitting large solvable groups of automorphisms." Adv. Math. 281 (2015):333-352.

Dixon, J. D., and Mortimer, B. Permutation Groups. Graduate Texts in Mathematics, 163. Springer-Verlag, New York, 1996.

Fima, P., Moon, S., and Stalder, Y. "Highly transitive actions of groups acting on trees." Proc. Amer. Math. Soc. 143 (2015):5083-5095.

Fima, P., Le Maître, F., Moon, S., and Stalder, Y. "High transitivity for more groups acting on trees." arXiv:2003.11116, 2020.

Freudenburg, G. Algebraic theory of locally nilpotent derivations. Second edition. Encyclopaedia of Mathematical Sciences, 136. Invariant Theory and Algebraic Transformation Groups, VII. Springer-Verlag, Berlin, 2017.

Garion, S. and Glasner, Y. "Highly transitive actions of $\operatorname{Out}\left(F_{n}\right)$." Groups Geom. Dyn. 7 (2013):357-376.

Gromov, M. "Groups of polynomial growth and expanding maps". With an appendix by Jacques Tits. Publ. Math. Inst. Hautes Études Sci. 53 (1981):53-73.

Fulton, W. Introduction to toric varieties. Annals of Mathematics Studies, 131. The William H. Roever Lectures in Geometry. Princeton University Press, Princeton, NJ, 1993.

Hochschild, G. P. Basic theory of algebraic groups and Lie algebras. Graduate Texts in Mathematics, 75. Springer-Verlag, New York-Berlin, 1981.

$\mathrm{Hu}$, Fei. "A theorem of Tits type for automorphism groups of projective varieties in arbitrary characteristic." With an appendix by Tomohide Terasoma. Math. Ann. (2019): https://doi.org/10.1007/s00208-019-01812-9.

Hull, M. and Osin, D. "Transitivity degrees of countable groups and acylindrical hyperbolicity." Israel J. Math. 216 (2016):307-353.

Humphreys, J. E. Linear Algebraic Groups. Graduate Texts in Mathematics, 21. Springer-Verlag, New YorkHeidelberg, 1975.

Jung, H. W. E. "Über ganze birationale Transformationen der Ebene." J. Reine Angew. Math. 184 (1942):161174.

Kambayashi, T. "On the absence of nontrivial separable forms of the affine plane." J. Algebra 35 (1975):449-456.

Kambayashi, T. "Automorphism group of a polynomial ring and algebraic group action on an affine space." J. Algebra 60 (1979):439-451.

Kramer, L. "Two-transitive Lie groups." J. Reine Angew. Math. 563 (2003):83-113.

Kurnosov, N., and Yasinsky, E. "Automorphisms of hypekähler manifolds and groups acting on CAT(0) spaces." arXiv:1810.09730, 2019.

Lamy, S. "L'alternative de Tits pour Aut( $\left.\mathbb{C}^{2}\right) . "$ J. Algebra 239 (2001):413-437.

Li, Y., Sauzin, D., and Sun, Sh. "The Baker-Campbell-Hausdorff formula via mould calculus." Lett. Math. Phys. 109 (2019):725--746.

Liendo, A. "G্a-actions of fiber type on affine T-varieties." J. Algebra 324 (2010):3653-3665.

Lewis, D., Perry, K., and Straub, A. "An algorithmic approach to the Polydegree Conjecture for plane polynomial automorphisms." J. Pure Appl. Algebra 223 (2019): 5346-5359.

Manetti, M. "The Baker-Campbell-Hausdorff formula." Notes of a course on deformation theory 2011-12. Available at: http://www1.mat.uniroma1.it/people/manetti/DT2011/BCHformula.pdf.

McDonough, T. P. "A permutation representation of a free group." Q. J. Math. 28 (1977):353-356.

Nagata, M. On automorphism group of $k[x, y]$. Department of Mathematics, Kyoto University, Lectures in Mathematics, No. 5. Kinokuniya Book-Store Co., Ltd., Tokyo, 1972. 
Oda, T. Convex bodies and algebraic geometry. An introduction to toric varieties. Ergebnisse der Mathematik und ihrer Grenzgebiete (3), 15. Springer-Verlag, Berlin, 1988.

Oguiso, K. "Tits alternative in hypekähler manifolds." Math. Res. Lett. 13 (2006):307-316.

Romaskevich, E. "Sums and commutators of homogeneous locally nilpotent derivations of fiber type." J. Pure Appl. Algebra 218 (2014):448-455.

Tits, J. "Sur les groupes doublement transitif continus." Comment. Math. Helv. 26 (1952):203-224.

Tits, J. "Correction et compléments" à: "Sur les groupes doublement transitif continus." Comment. Math. Helv. 30 (1956):234-240

Tits, J. "Free subgroups in linear groups." J. Algebra 20 (1972): 250-270.

Urech, Ch. "Subgroups of elliptic elements of the Cremona group." J. Reine Angew. Math. 000010151520200008. Published online: 15 May 2020.

van der Kulk, W. "On polynomial rings in two variables." Nieuw Arch. Wiskd. (5) 1 (1953):33-41.

Wang, L., Gao, Y., and Jing, N. "On multivariable Zassenhaus formula." Front. Math. China 14 (2019):421-433.

Whittaker, J. V. "Multiply transitive groups of transformations." Pacific J. Math. 23 (1967):189-207.

Wikipedia. "Ping-pong lemma". Available at: https : //en.wikipedia.org/wiki/Ping - pong_lemma.

Wright, D. "Algebras which resemble symmetric algebra." Ph. D. thesis. Columbia University, 1975. 